Supporting Information for

\title{
Reversible Switching of Molecular Conductance in Viologens is Controlled by the Electrochemical Environment
}

Jialing Li ${ }^{1,4,5}$, Sanja Pudar ${ }^{2,5}$, Hao Yu ${ }^{1,5}$, Songsong Li ${ }^{3,4,5}$, Jeffrey S. Moore ${ }^{2,3,4,5}$, Joaquín Rodríguez-López ${ }^{2,4,5}$, Nicholas E. Jackson ${ }^{2}$, Charles M. Schroeder ${ }^{1,2,3,4,5 *}$

${ }^{1}$ Department of Chemical and Biomolecular Engineering, University of Illinois at UrbanaChampaign, Urbana, Illinois, 61801, United States

${ }^{2}$ Department of Chemistry, University of Illinois at Urbana-Champaign, Urbana, Illinois, 61801, United States

${ }^{3}$ Department of Materials Science and Engineering, University of Illinois at Urbana-Champaign, Urbana, Illinois, 61801, United States

${ }^{4}$ Beckman Institute for Advanced Science and Technology, University of Illinois at UrbanaChampaign, Urbana, Illinois, 61801, United States

${ }^{5}$ Joint Center for Energy Storage Research (JCESR), Argonne, Illinois 60439, United States

*To whom correspondence should be addressed. Email: cms@illinois.edu

Table of Contents:

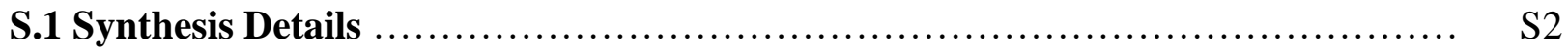

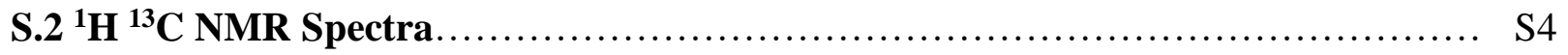

S.3 Supplementary STM-BJ Experimental Data .................................. S6

S.4 Supplementary Molecular Modeling of 2+ State ............................ S10

S.5 Supplementary Bulk Electrochemical and UV-Vis Characterization Data .......... S14

S.6 Supplementary ECSTM-BJ Experimental Data with Electrochemical Gating...... S17

S.7 Supplementary Molecular Modeling of Radical Cationic State.....................S19

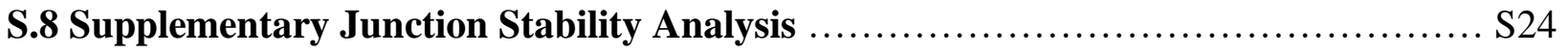




\section{S.1 Synthesis Details}

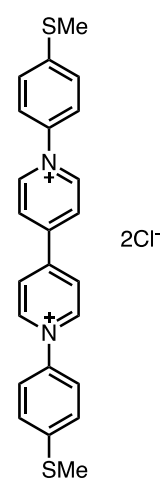

1,1'-bis(4-(methylthio)phenyl)-[4,4'-bipyridine]-1,1'-diium chloride (DTPV $\left.{ }^{2+}-\mathrm{Cl}^{-}\right)$. DTPV $^{2+}-\mathbf{C l}^{-}$is synthesized according to literature procedures. ${ }^{1} 1,1^{\prime}$-bis $(2,4-$ dinitrophenyl)-[4,4'-bipyridine]-1,1'-diium chloride $(0.50 \mathrm{~g}, 0.89 \mathrm{mmol})$ and 4(methylthio)aniline $(0.67 \mathrm{~g}, 4.8 \mathrm{mmol})$ were refluxed in ethanol $(150 \mathrm{~mL})$ overnight. The solvent was concentrated under vacuum and poured into THF. The orange precipitate was collected by filtration and washed with cold DCM to obtain the product as orange solid. $(0.2 \mathrm{~g}, 47 \%)$. Characterization data matched previously reported literature. Following the previously reported procedures ${ }^{2}, \mathbf{D T P V}^{2+} \mathbf{P F}_{6}$, $\mathbf{D T P V}^{2+}$ $\mathbf{C l O}_{4}^{-}$, DTPV $^{2+}-\mathbf{B F}_{4}^{-}$were prepared by adding saturated ammonium hexafluorophosphate, lithium perchlorate, and ammonium tetrafluoroborate aqueous solution to a solution of $\mathbf{D T P V}^{2+}-\mathbf{C l}$. The resulting solids were collected by filtration and dried under reduced pressure, which were used without further purification and characterization. $\mathbf{D T P V}^{\mathbf{2}}-\mathbf{B r}^{-}$and DTPV $^{2+}$-I $^{-}$were prepared by adding diluted aqueous solution of $\mathrm{HBr}$ and $\mathrm{HI}$ to an acetone solution of DTPV $^{2+} \mathbf{P F}_{6}$. The resulting solids were collected by filtration and dried under reduced pressure, which were used without further purification and characterization.
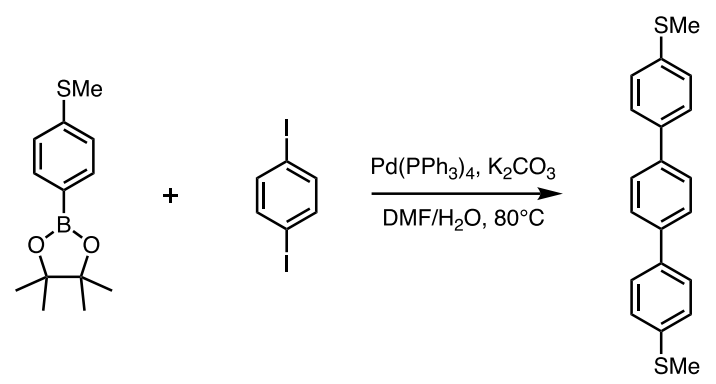

4,4"-bis(methylthio)-1,1':4',1"-terphenyl (DTP). To a flame-dried flask was added 4(methylthio)phenylboronic acid pinacolate $(100 \mathrm{mg}$, $0.43 \mathrm{mmol}), 1,4-D i i o d o b e n z e n e ~(70 \mathrm{mg}, 0.21 \mathrm{mmol})$, $\mathrm{Pd}\left(\mathrm{PPh}_{3}\right)_{4}(4 \mathrm{mg}, 0.0043 \mathrm{mmol}), \mathrm{K}_{2} \mathrm{CO}_{3}(233 \mathrm{mg}, 1.69$ $\mathrm{mmol})$, degassed DMF $(10 \mathrm{~mL})$, and $\mathrm{H}_{2} \mathrm{O}(0.5 \mathrm{~mL})$. The reaction was heated at $80^{\circ} \mathrm{C}$ for 2 days. After cooling down to room temperature, the mixture was poured into water $(100 \mathrm{~mL})$ and stirred for 1 $\mathrm{hr}$. The reaction mixture was filtered, and the precipitate was washed with water and THF. The precipitate was dried under vacuum to afford product as white solid (50 mg, 74\%). Characterization data matched literature. ${ }^{3}{ }^{1} \mathrm{H}$ NMR $\left(500 \mathrm{MHz}, \mathrm{CDCl}_{3}\right) \delta 7.64(\mathrm{~s}, 4 \mathrm{H}), 7.57(\mathrm{~d}, J=$ $8.4 \mathrm{~Hz}, 4 \mathrm{H}), 7.35$ (d, $J=8.3 \mathrm{~Hz}, 4 \mathrm{H}), 2.54$ (s, 6H).

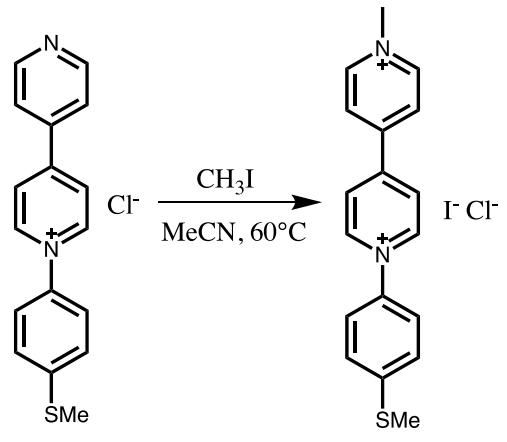

1-methyl-1'-(4-(methylthio)phenyl)-[4,4'-bipyridine]-1,1'diium iodide chloride $\left(\mathbf{T P V}^{2+} \mathbf{I}^{-}, \mathbf{C l}^{-}\right)$. To an Ace pressure tube was added 4-pyridyl)pyridinium chloride (100 $\mathrm{mg}, 0.32 \mathrm{mmol})$, methyl iodide $(452 \mathrm{mg}, 3.2 \mathrm{mmol})$, and acetonitrile $(5 \mathrm{~mL})$. The reaction was heated at $60^{\circ} \mathrm{C}$ overnight. After cooling down to room temperature, the mixture was poured into THF $(100 \mathrm{~mL})$ and stirred for $1 \mathrm{hr}$. The reaction mixture was filtered, and the precipitate was washed with THF and DCM. The precipitate was dried under vacuum to afford product as orange solid (60 mg, 41\%). ${ }^{1} \mathrm{H}$ NMR (500 MHz, DMSO) 
$\delta 9.64(\mathrm{~d}, J=6.7 \mathrm{~Hz}, 2 \mathrm{H}), 9.32(\mathrm{~d}, J=6.3 \mathrm{~Hz}, 2 \mathrm{H}), 8.94(\mathrm{~s}, 1 \mathrm{H}), 8.93-8.85(\mathrm{~m}, 3 \mathrm{H}), 7.90(\mathrm{~d}, J=$ $8.6 \mathrm{~Hz}, 2 \mathrm{H}), 7.65(\mathrm{~d}, J=8.8 \mathrm{~Hz}, 2 \mathrm{H}), 4.47(\mathrm{~s}, 3 \mathrm{H}), 2.62(\mathrm{~s}, 3 \mathrm{H}) .{ }^{13} \mathrm{C}$ NMR $(125 \mathrm{MHz}, \mathrm{DMSO}) \delta$ 149.40, 148.24, 147.18, 146.05, 144.12, 139.26, 126.91, 126.86, 126.69, 125.57, 48.57, 14.89. TPV $^{2+} \mathbf{P F}_{\mathbf{6}}$ was prepared by adding saturated ammonium hexafluorophosphate and ammonium tetrafluoroborate aqueous solution to the solution of $\mathbf{T P V}^{2+}-\mathbf{I}$; $\mathbf{C l}$. The resulting solids were collected by filtration and dried under reduced pressure, which were used without further purification and characterization.

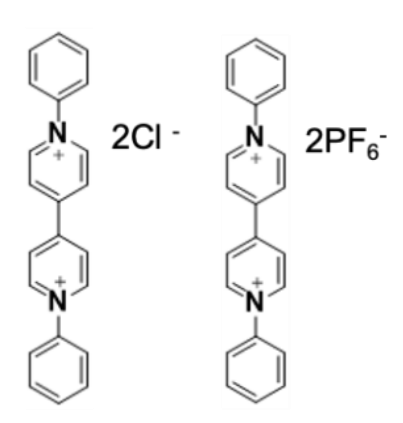

1,1'-diphenyl-[4,4'-bipyridine]-1,1'-diium chloride $\left(\mathrm{DPV}^{2+}-\mathrm{Cl}^{-}\right)$is synthesized according to previous reports. ${ }^{4}$ Characterization data matched prior literature. $\mathbf{D P V}{ }^{2+}{ }_{-} \mathbf{P F}_{\mathbf{6}}{ }^{-}$was prepared by adding saturated ammonium hexafluorophosphate and ammonium tetrafluoroborate aqueous solution to the solution of $\mathbf{D P V}^{2+}-\mathbf{C l}^{\mathbf{l}}$. The resulting solids were collected by filtration and dried under reduced pressure, which were used without further purification and characterization.

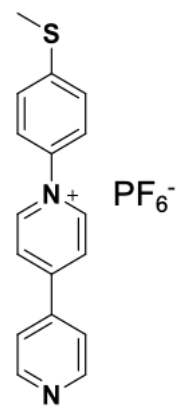

1-(4-(Methylthio)phenyl)-[4,4'-bipyridin]-1-ium $\left(\mathbf{T P P}^{+}-\mathrm{Cl}^{-}\right)$is synthesized following previously reported procedures. ${ }^{5} \mathbf{T P P}^{+}-\mathbf{P F}_{\mathbf{6}}{ }^{-}$was prepared by adding saturated ammonium hexafluorophosphate aqueous solution to a solution of $\mathbf{T P P}^{+}$-Cl- The resulting solids were collected by filtration and dried under reduced pressure, which were used without further purification and characterization. 


\section{S.2 ${ }^{1} \mathrm{H}^{13} \mathrm{C}$ NMR Spectra}

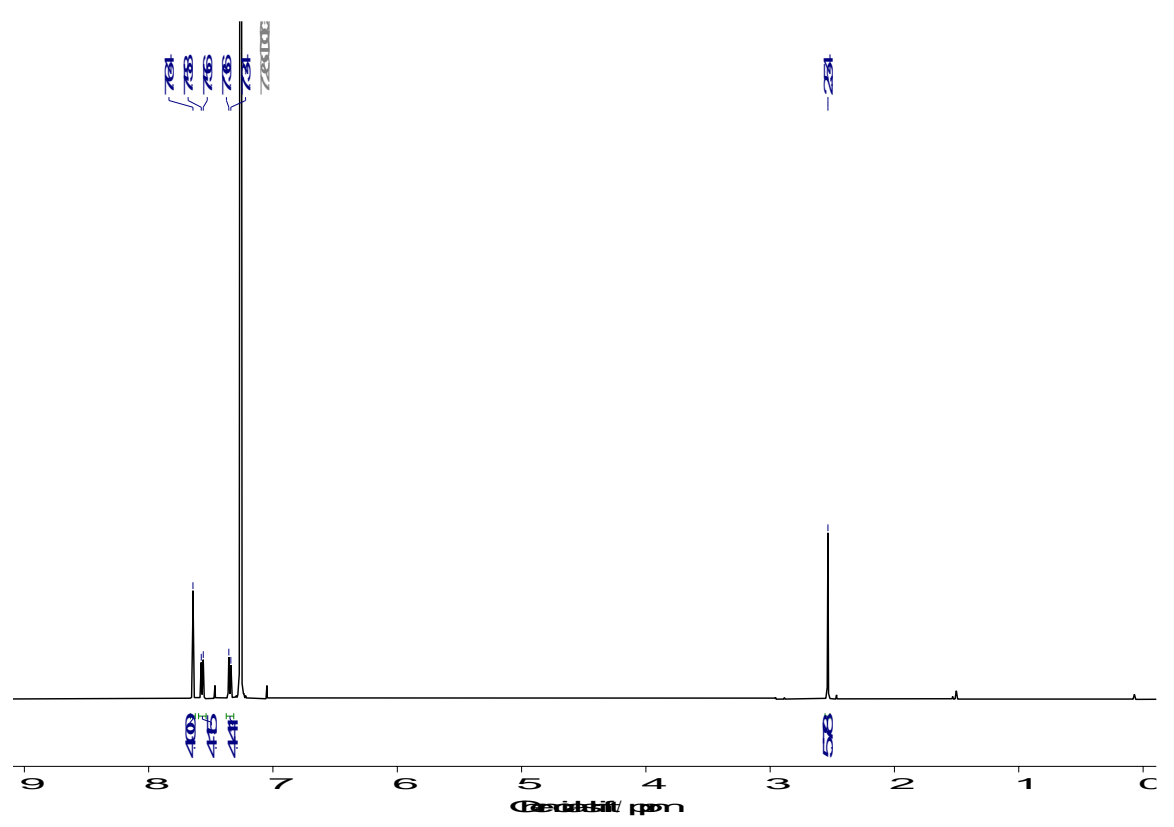

Figure S1. ${ }^{1} \mathrm{H}$ NMR spectrum of DTP $\left(500 \mathrm{MHz}, \mathrm{CDCl}_{3}\right)$.

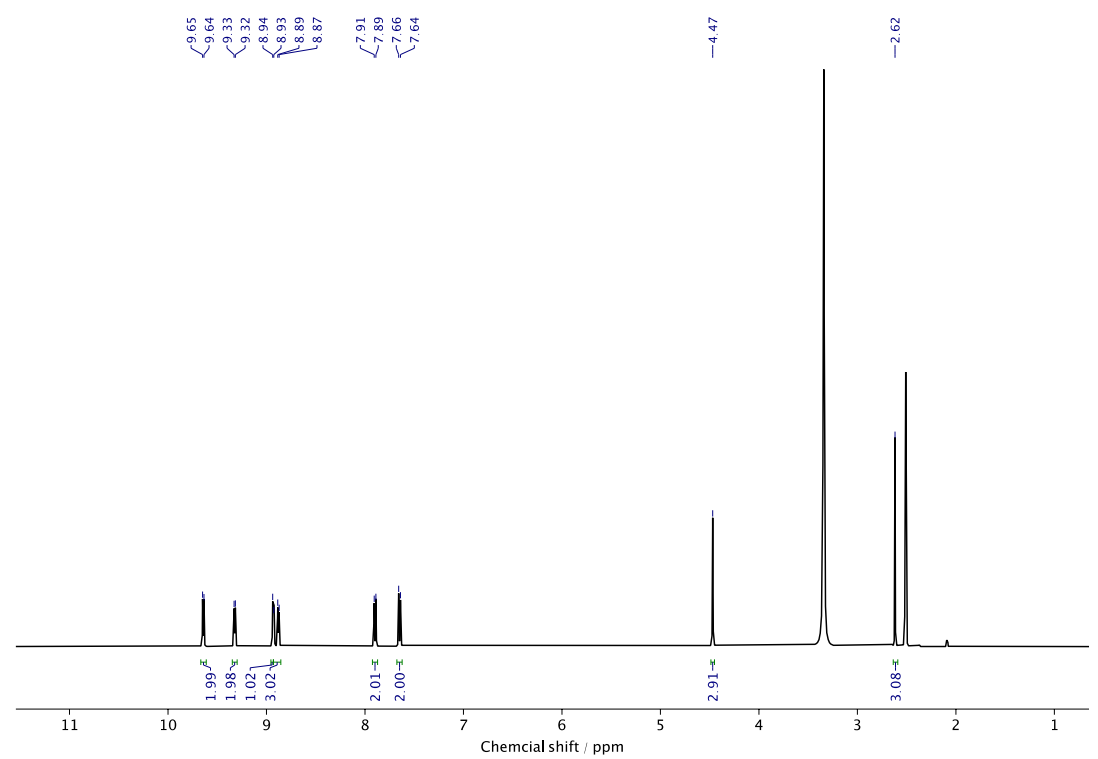

Figure S2. ${ }^{1} \mathrm{H}$ NMR spectrum of $\mathbf{T P V}^{2+}-\mathbf{I}^{-}, \mathbf{C l}^{-}\left(500 \mathrm{MHz}, \mathrm{CDCl}_{3}\right)$. 


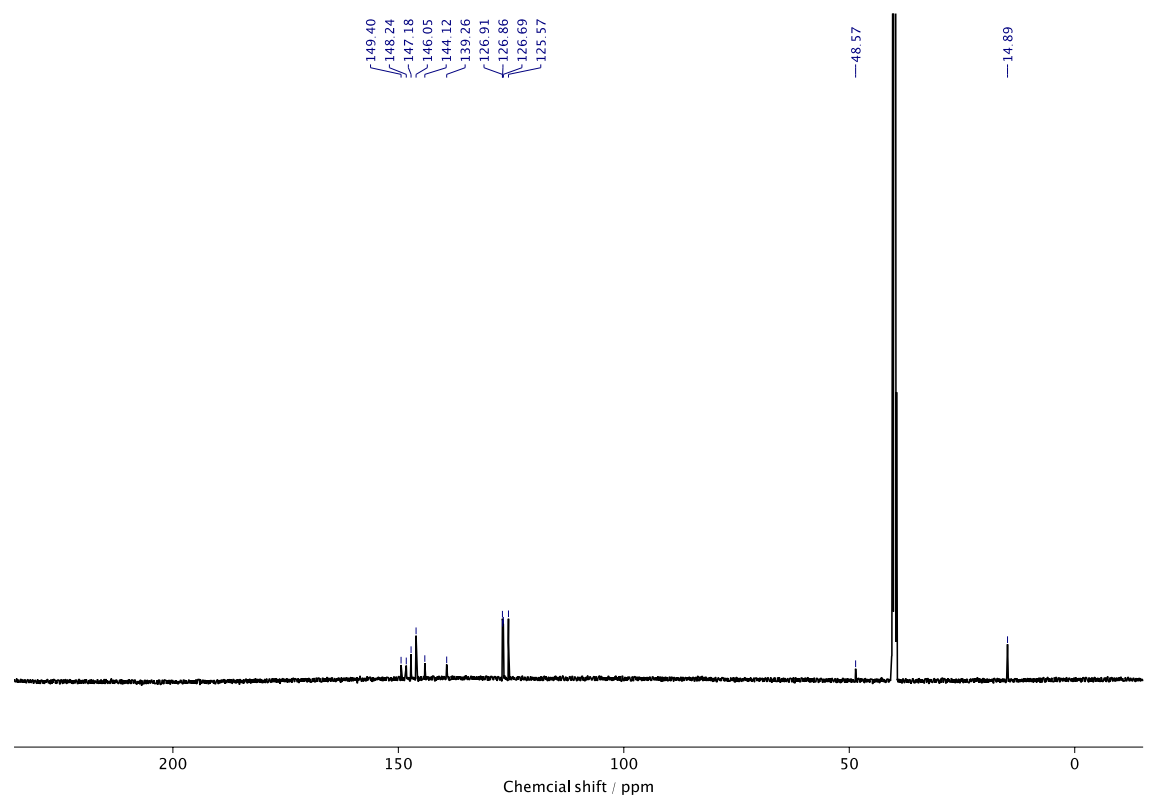

Figure S3. ${ }^{13} \mathrm{C}$ NMR spectrum of $\mathbf{T P V}^{2+}-\mathbf{I}^{-}, \mathbf{C l}^{-}\left(500 \mathrm{MHz}, \mathrm{CDCl}_{3}\right)$. 


\section{S.3 Supplementary STM-BJ Experimental Data}

(a)

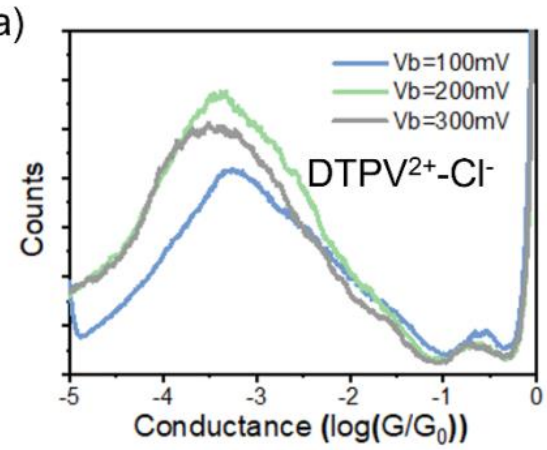

(c)

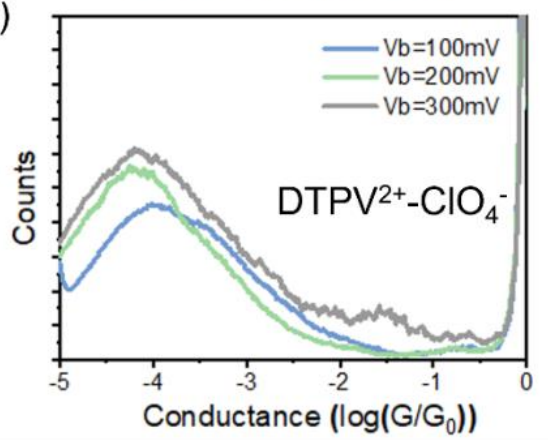

(b)

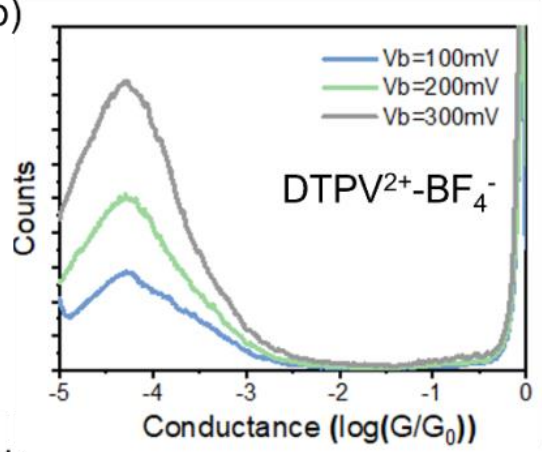

(d)

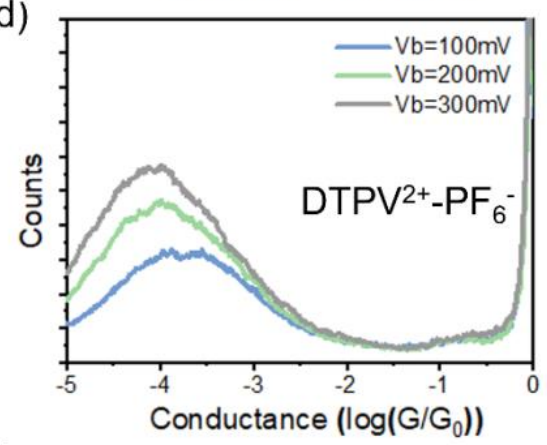

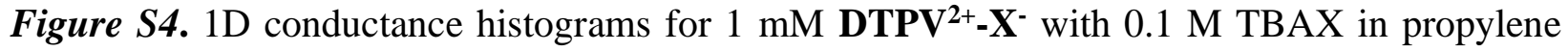
carbonate (PC) at $100 \mathrm{mV}, 200 \mathrm{mV}$, and $300 \mathrm{mV}$ without applying electrochemical gate potential. $\mathrm{X}=$ (a) $\mathrm{Cl}^{-}$; (b) $\mathrm{BF}_{4}^{-}$; (c) $\mathrm{ClO}_{4}^{-}$; (d) $\mathrm{PF}_{6}^{-}$. 
(a)
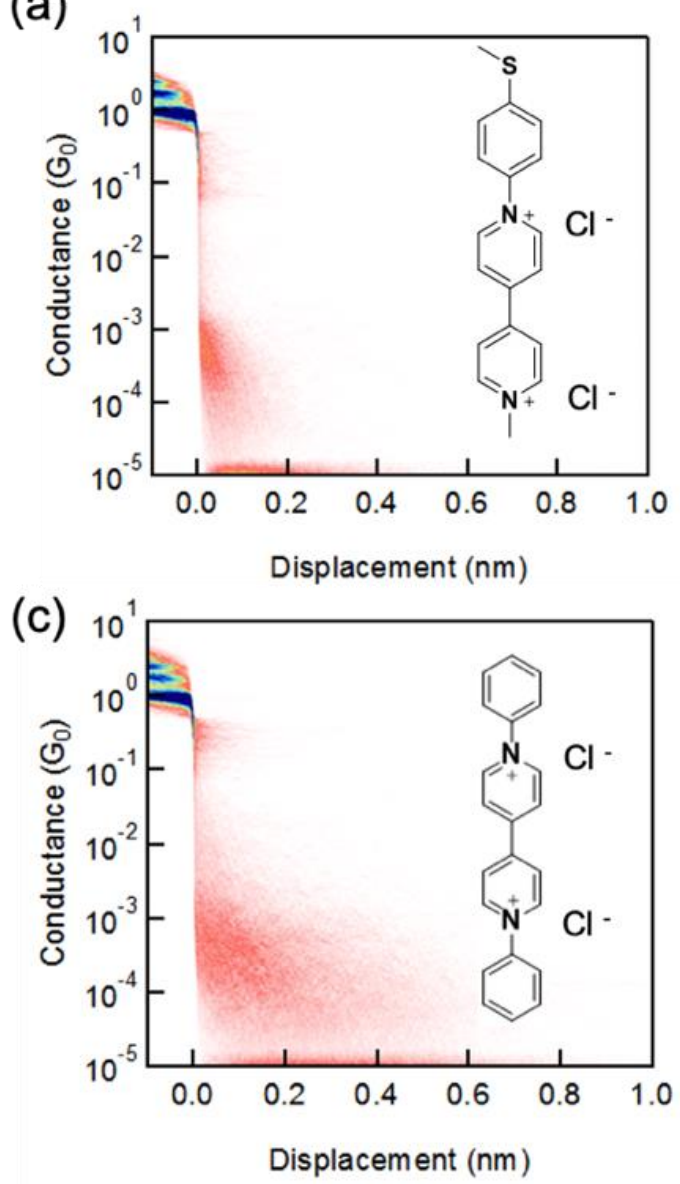

(b)

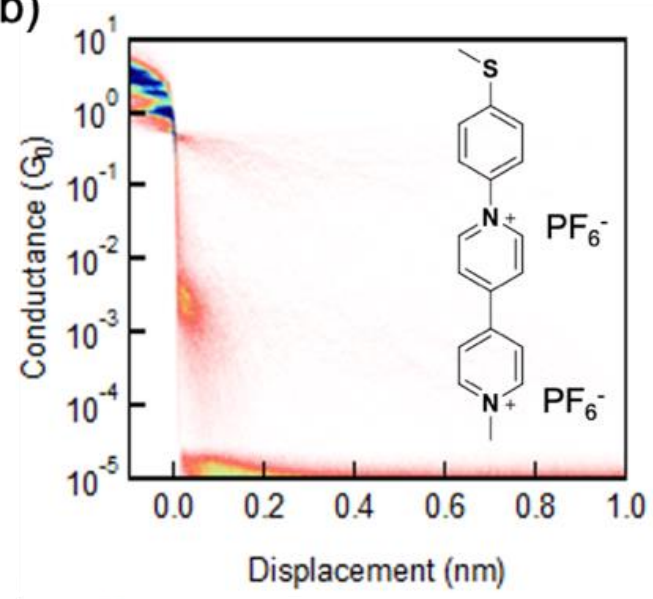

(d)

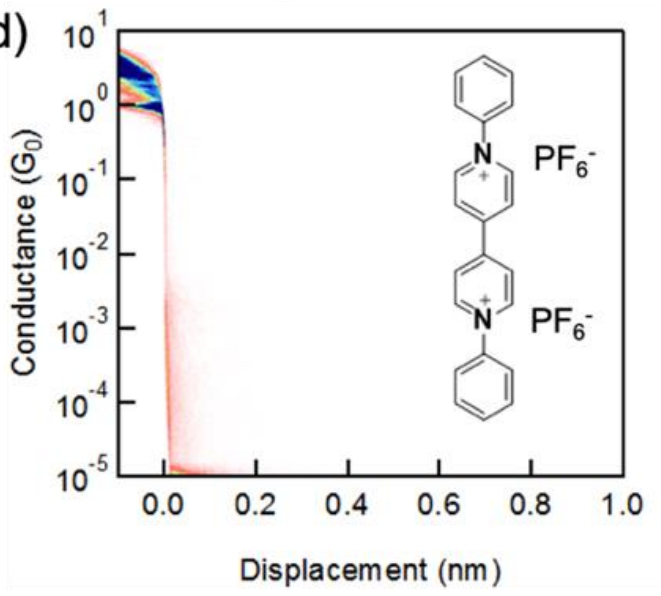

Figure S5. 2D conductance-displacement histograms for (a) $1 \mathrm{mM} \mathrm{TPV}^{\mathbf{2 +}} \mathbf{C l}^{-}$with $0.1 \mathrm{M} \mathrm{TBACl}$; (b) $1 \mathrm{mM}$ TPV ${ }^{2+}-\mathbf{P F}_{6}{ }^{-}$with $0.1 \mathrm{M} \mathrm{TBAPF}_{6}$; (c) $1 \mathrm{mM} \mathrm{DPV}^{2+}-\mathbf{C l}^{-}$with $0.1 \mathrm{M} \mathrm{TBACl}$; (d) $1 \mathrm{mM}$ DPV $^{2+}-$ PF $_{6}-$ with $0.1 \mathrm{M} \mathrm{TBAPF}_{6}$ in $\mathrm{PC}$ at $100 \mathrm{mV}$ without electrochemical gate potential. 

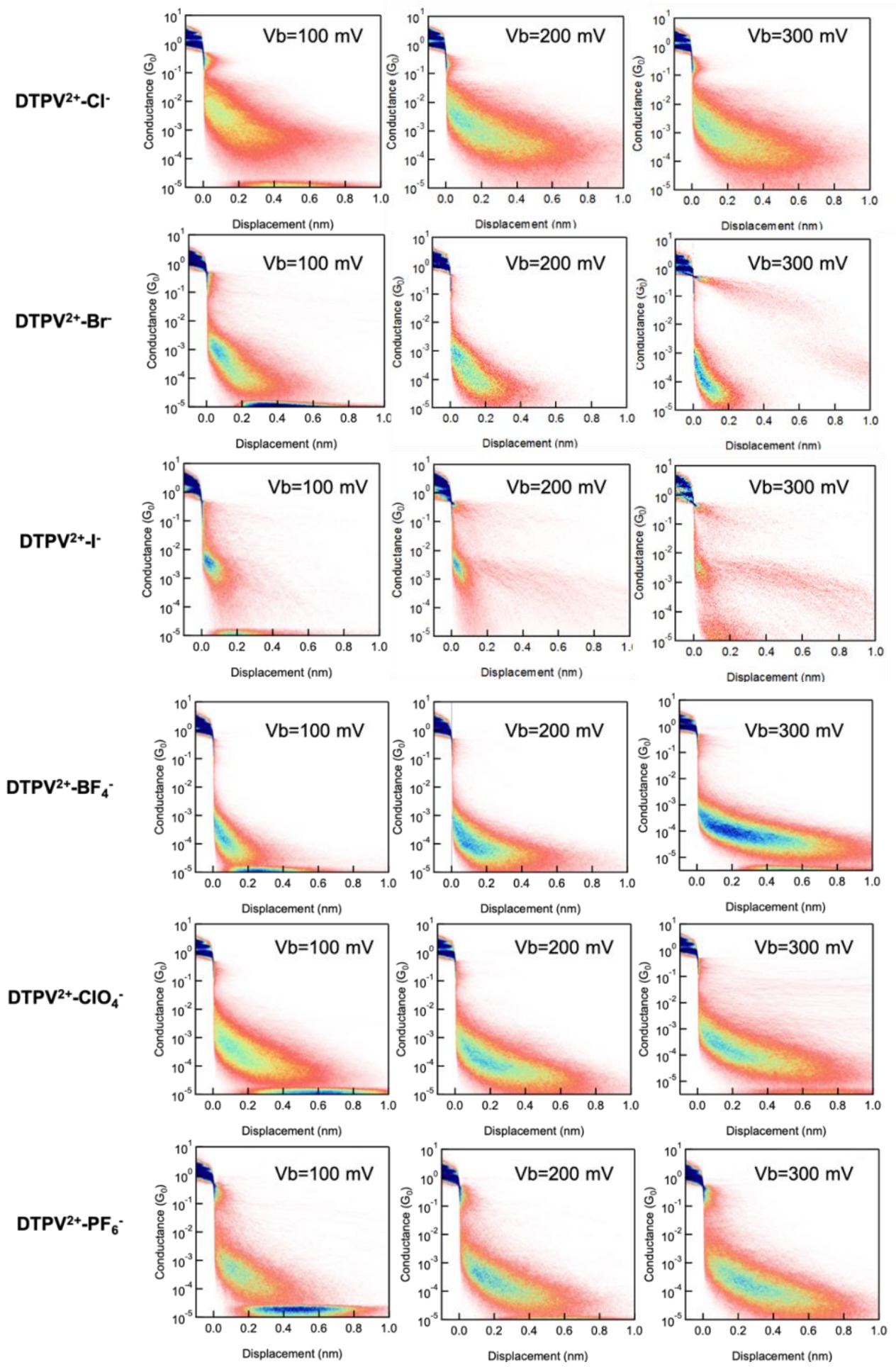

Figure S6. 2D conductance-displacement histograms for $1 \mathrm{mM} \mathrm{DTPV}^{2+}-\mathbf{X}$ with $0.1 \mathrm{M}$ TBAX in propylene carbonate (PC) at $100 \mathrm{mV}, 200 \mathrm{mV}, 300 \mathrm{mV}$ without electrochemical gate potential. 1st row: $\mathbf{D T P V}^{2+} \mathbf{C l}^{-}$, 2nd row: $\mathbf{D T P V}^{2+}-\mathbf{B r}^{-}, 3$ rd row: $\mathbf{D T P V}^{2+}-\mathbf{I}^{-}$, 4th row: $\mathbf{D T P V}^{2+}-\mathbf{B F}_{4}{ }^{-}$, 5th row: DTPV $^{2+}-\mathrm{ClO}_{4}{ }^{-}, 6^{\text {th }}$ row: $\mathbf{D T P V}^{2+}-\mathbf{P F}_{6}$. Each data point shown in Figure $1 \mathrm{~b}$ (main text) 
corresponds to the ratio of peak conductance values of $2 \mathrm{D}$ conductance-displacement histograms containing 5,000-10,000 single molecule conductance traces.

(a)

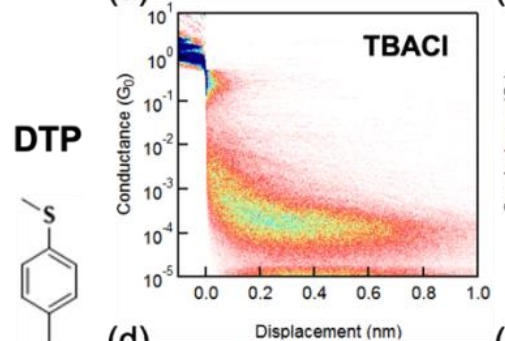

(d)

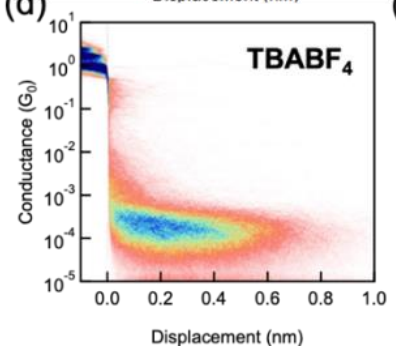

(b)

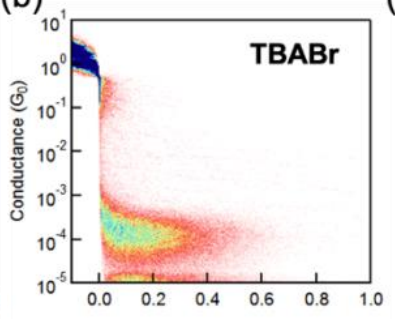

(c)

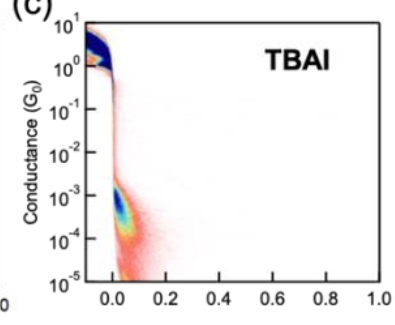

(e)
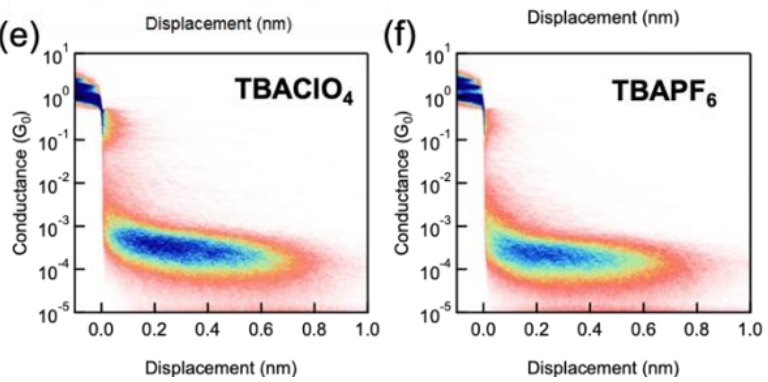

Figure S7. 2D conductance-displacement histograms for $0.1 \mathrm{mM}$ DTP with $10 \mathrm{mM}$ (a) TBACl; (b) TBABr; (c) TBAI; (d) $\mathrm{TBABF}_{4}$; (e) $\mathrm{TBAClO}_{4}$; (f) $\mathrm{TBAPF}_{6}$ in PC at $200 \mathrm{mV}$ tip-to-substrate voltage. For these experiments, a concentration of $0.1 \mathrm{mM}$ DTP was used due to relatively low solubility in propylene carbonate.

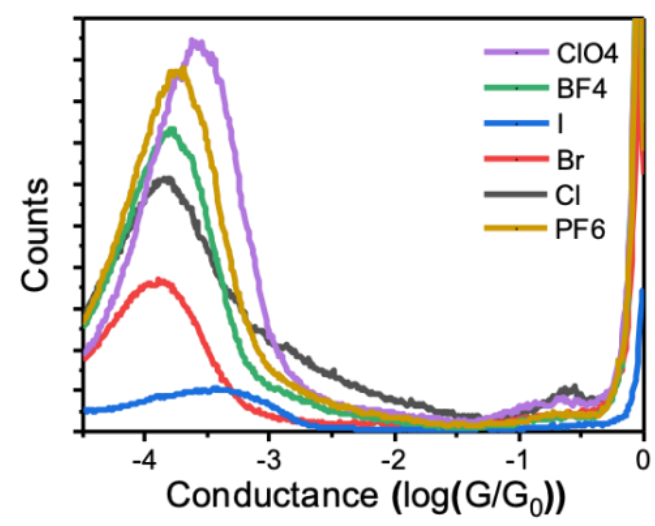

Figure S8. 1D conductance histograms for $0.1 \mathrm{mM}$ DTP with $10 \mathrm{mM}$ TBACl (black); TBABr (red); TBAI (blue); $\mathrm{TBABF}_{4}$ (green); $\mathrm{TBAClO}_{4}$ (purple); $\mathrm{TBAPF}_{6}$ (yellow) in $\mathrm{PC}$ at $100 \mathrm{mV}$ tipto-substrate voltage. 


\section{S.4 Supplementary Molecular Modeling of 2+ state}

S.4.1 Geometry Optimization and Electronic Structure Analysis by Density Functional Theory (DFT)

For $\mathrm{Cl}^{-}$counterions, numerous conformational minima were identified from the conformational search procedure. Table S1 shows the result of the conformational search. For each geometry there is an approximate degeneracy associated the positions of the counterions in the minimum energy geometry. These approximate degeneracies were used in computing the Boltzmann averaged values of HOMO energies, LUMO energies, and end-to-end distances.

Table S1. Results of conformational minima of $\mathbf{D T P V}^{2+}{ }_{-} \mathrm{Cl}^{-}$

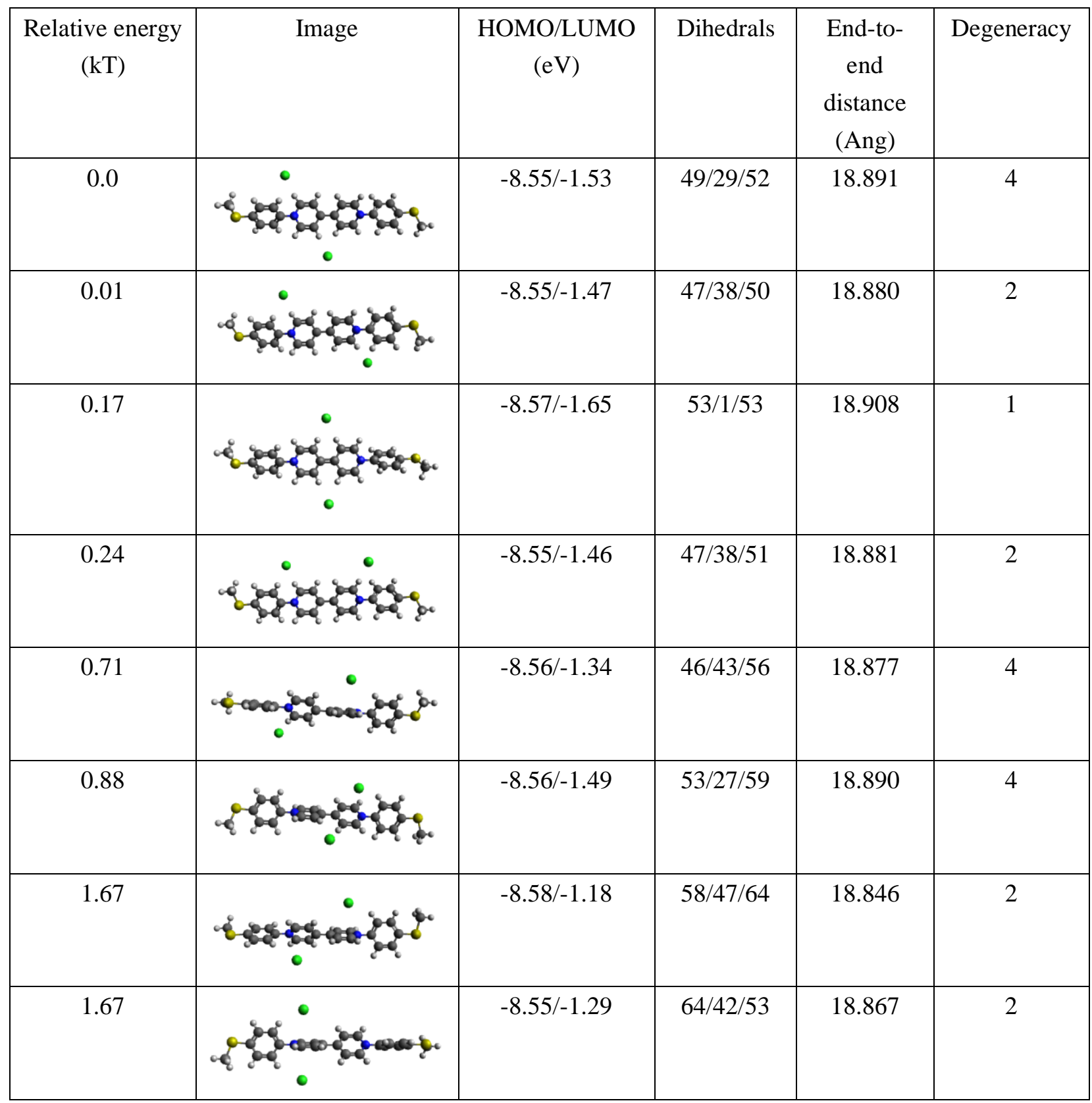


For $\mathrm{BF}_{4}^{-}$counterions, two distinct conformational minima were identified from the conformational search procedure. Table S2 shows the result of the conformational search.

Table S2. Results of conformational minima of $\mathbf{D T P V}^{2+}{ }_{-} \mathbf{B F}_{4}{ }^{-}$

\begin{tabular}{|c|c|c|c|c|c|}
\hline $\begin{array}{l}\text { Relative } \\
\text { energy (kT) }\end{array}$ & Image & $\begin{array}{l}\text { HOMO/LUMO } \\
(\mathrm{eV})\end{array}$ & Dihedrals & $\begin{array}{l}\text { End-to- } \\
\text { end } \\
\text { distance } \\
\text { (Ang) }\end{array}$ & Degeneracy \\
\hline 0.0 & & $-8.58 /-1.31$ & $55 / 43 / 53$ & 18.862 & 2 \\
\hline 0.78 & & $-8.58 /-1.25$ & $58 / 44 / 55$ & 18.867 & 2 \\
\hline
\end{tabular}

For $\mathrm{ClO}_{4}^{-}$counterions, two distinct conformational minima were identified from the conformational search procedure. Table S3 shows the result of the conformational search.

Table S3. Results of conformational minima of DTPV $^{2+}$ ClOH$_{4}^{-}$

\begin{tabular}{|c|c|c|c|c|c|}
\hline $\begin{array}{c}\text { Relative } \\
\text { energy (kT) }\end{array}$ & Image & $\begin{array}{c}\text { HOMO/LUMO } \\
(\mathrm{eV})\end{array}$ & Dihedrals & $\begin{array}{l}\text { End-to- } \\
\text { end } \\
\text { distance } \\
\text { (Ang) }\end{array}$ & Degeneracy \\
\hline 0.0 & 9 & $-8.57 /-1.29$ & $58 / 45 / 54$ & 18.867 & 2 \\
\hline 0.78 & & $-8.58 /-1.26$ & $53 / 47 / 56$ & 18.843 & 2 \\
\hline
\end{tabular}


For $\mathrm{PF}_{6}-$ counterions, two distinct conformational minima were identified from the conformational search procedure. Table S4 shows the result of the conformational search.

Table S4. Results of conformational minima of $\mathbf{D T P V}^{2+} \mathbf{P F}_{\mathbf{6}} \mathbf{}^{-}$

\begin{tabular}{|c|c|c|c|c|c|}
\hline $\begin{array}{c}\text { Relative } \\
\text { energy (kT) }\end{array}$ & Image & $\begin{array}{c}\text { HOMO/LUMO } \\
(\mathrm{eV})\end{array}$ & Dihedrals & $\begin{array}{c}\text { End-to- } \\
\text { end } \\
\text { distance } \\
\text { (Ang) }\end{array}$ & Degeneracy \\
\hline 0.0 & & $-8.59 /-1.39$ & $54 / 40 / 54$ & 18.867 & 2 \\
\hline 0.78 & & $-8.58 /-1.37$ & $55 / 42 / 50$ & 18.867 & 2 \\
\hline
\end{tabular}


S.4.2 DFT-based transmission calculations

(a)

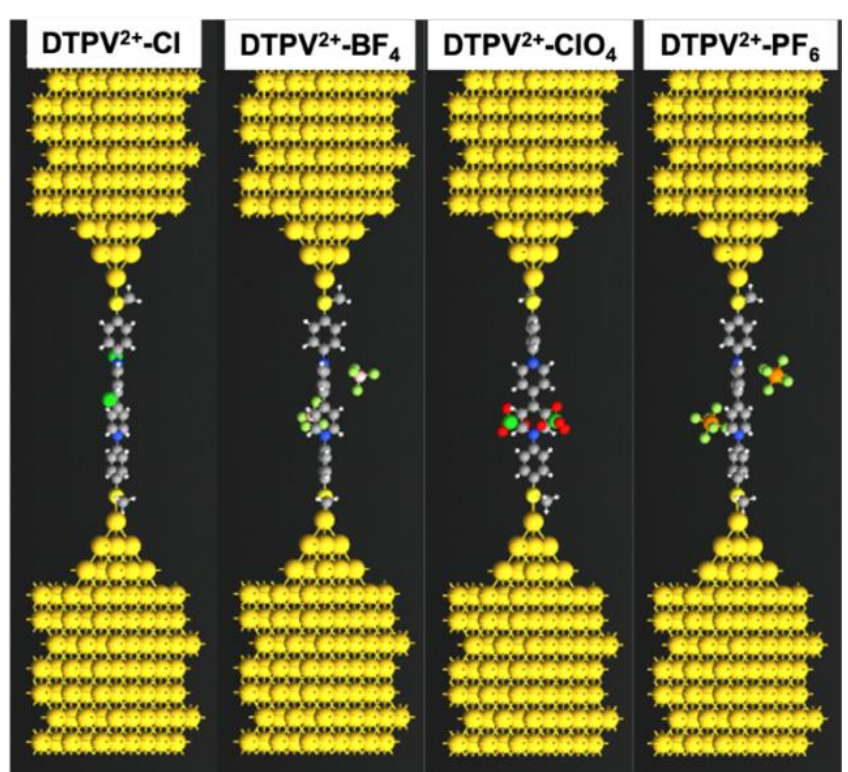

(b)

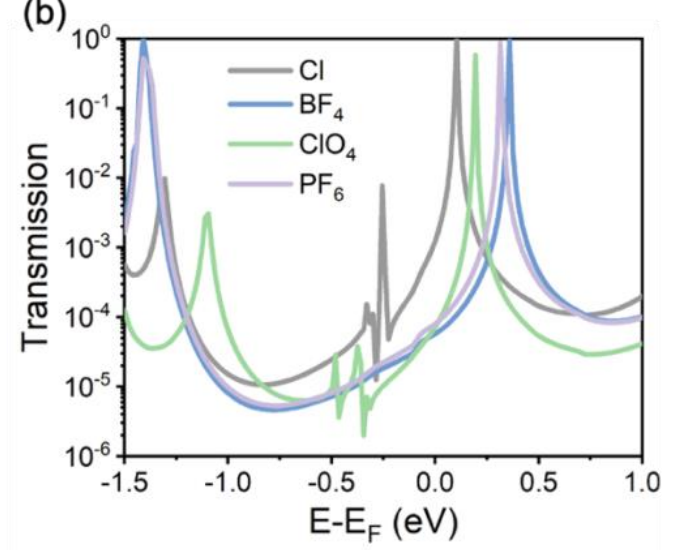

Figure S9. Nonequilibrium Green's function-density functional theory (NEGF-DFT) simulations

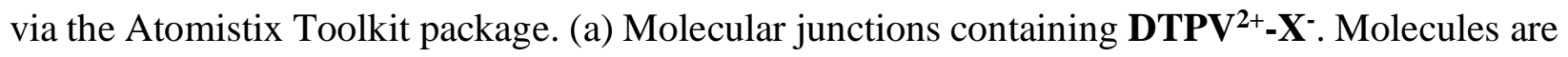
in the dicationic (+2) state. (b) Transmission as a function of energy for $\mathbf{D T P V}^{\mathbf{2 +}}-\mathbf{X}^{-} . E_{F}$ is the Fermi level of the junction. 


\section{S.5 Supplementary Bulk Electrochemical and UV-Vis Characterization Data}
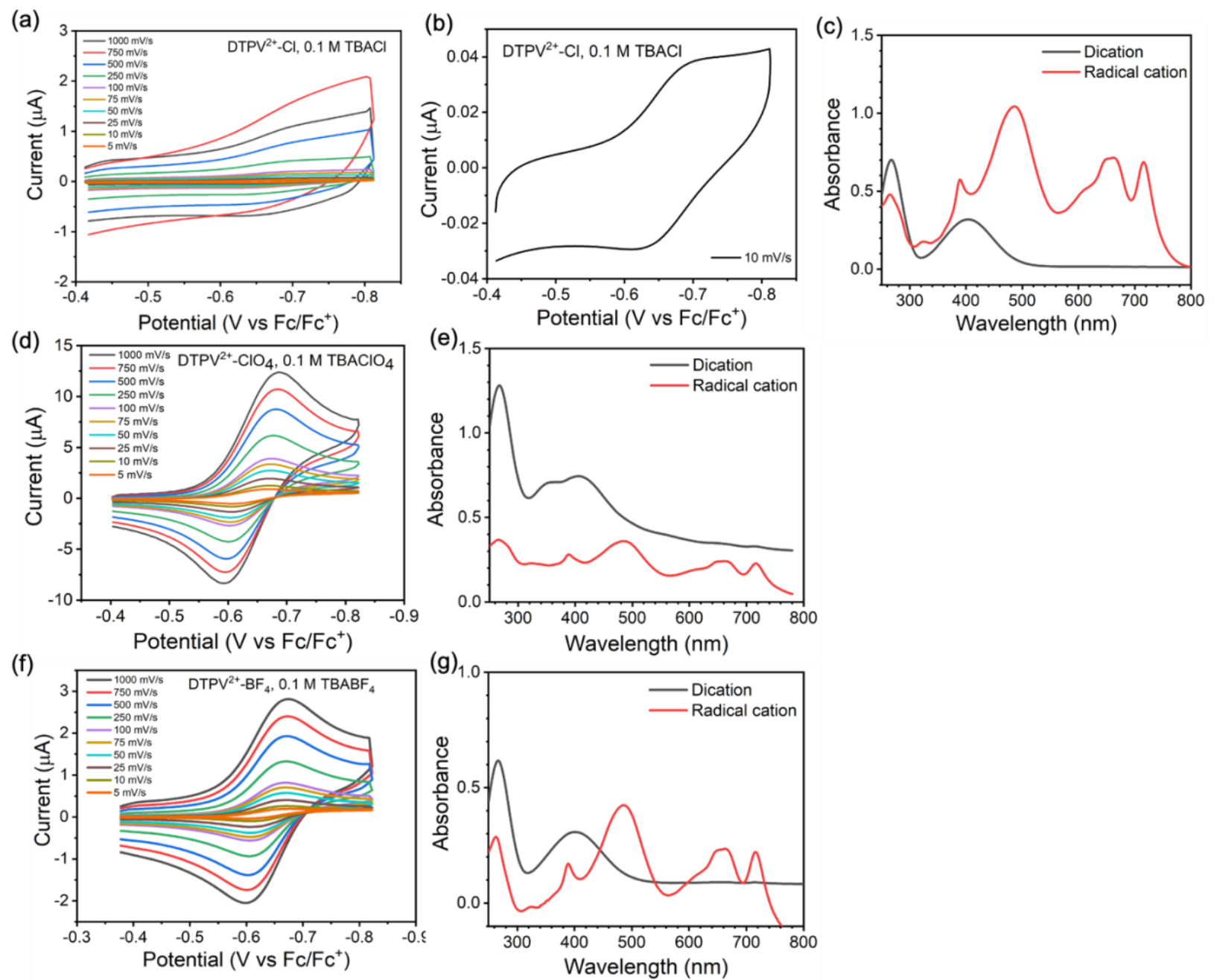

Figure S10. Scan-rate dependence of (a)-(b) $1.2 \mathrm{mM} \mathrm{DTPV}^{2+}-\mathrm{Cl}^{-}$; (d) $1.1 \mathrm{mM} \mathrm{DTPV}^{2+}-\mathrm{ClO}_{4}^{-}$; (f) $0.57 \mathrm{mM} \mathrm{DTPV}^{2+}-\mathbf{B F}_{4}{ }^{-}$; UV-vis spectra of initial and reduced states of $\mathbf{D T P V}^{2+}$ species with anions of (c) $\mathbf{C l}^{-}$; (e) $\mathbf{C l O}_{4}^{-;}$; (g) $\mathbf{B F}_{4}^{-;}$; All cyclic voltammetry was performed on Au macroelectrode (area of $0.03 \mathrm{~cm}^{2}$ ); All conditions were in $0.1 \mathrm{M}$ TBAX, propylene carbonate, unless otherwise stated. Scan-rate dependent experiments were performed using a polypyrrole quasi reference electrode, which was manufactured by coating Pt wire with partially oxidized polypyrrole. The quasi-reference electrode was stored in a glass tube containing $0.1 \mathrm{M}$ TBAX in PC, fitted with a frit at one end. 
(a)

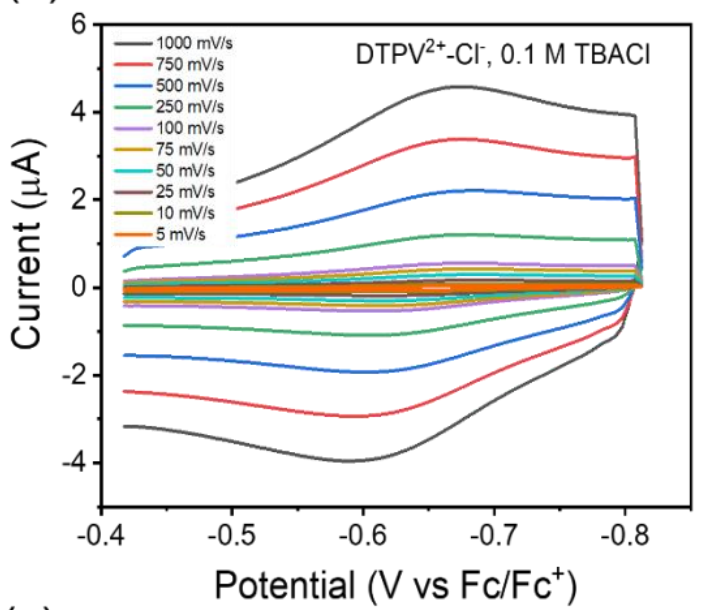

(c)

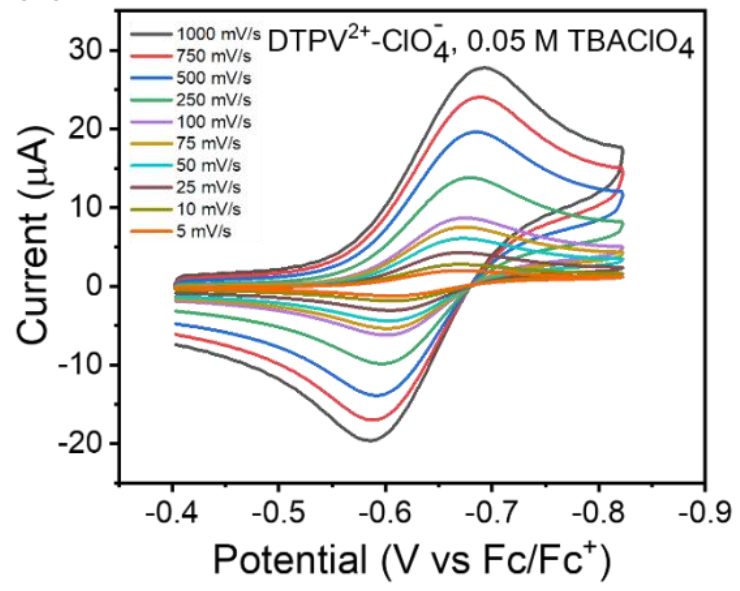

(b)

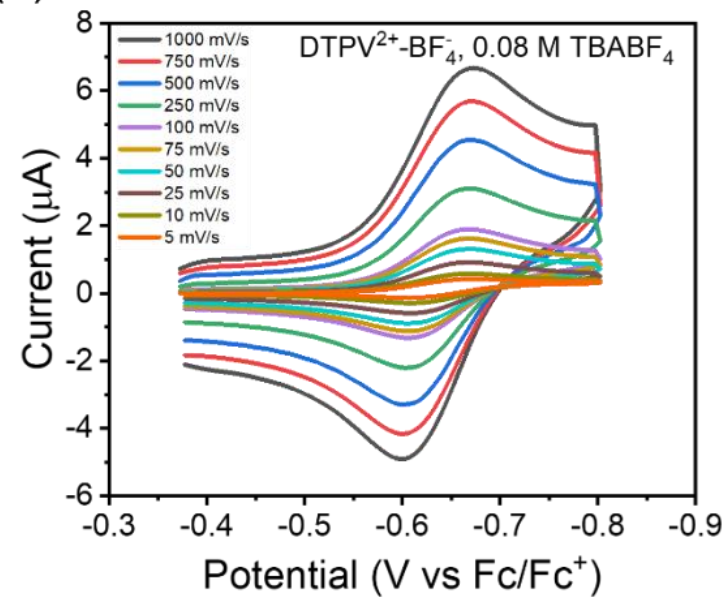

(d)

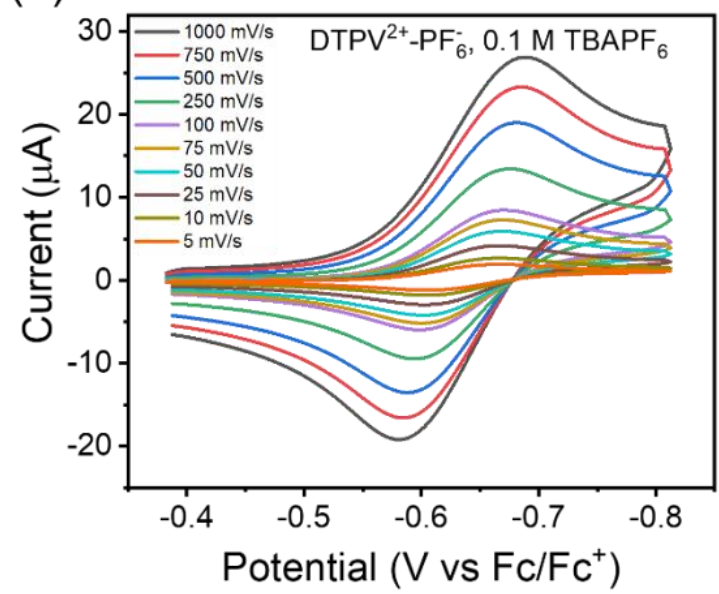

Figure S11. Scan-rate dependence of (a) $\mathbf{D T P V}^{2+}-\mathrm{Cl}^{-}$; (b) $\mathbf{D T P V}^{2+}-\mathrm{BF}_{4}^{-}$; (c) $\mathbf{D T P V}^{2+}-\mathrm{ClO}_{4}^{-}$; (d) DTPV $^{2+}-\mathbf{P F}_{6}$. In these experiments, cyclic voltammetry was performed on glassy carbon macroelectrodes (area of $0.07 \mathrm{~cm}^{2}$ ). Scan-rate dependent experiments were performed using a polypyrrole quasi reference electrode, which was manufactured by coating Pt wire with partially oxidized polypyrrole. The quasi-reference electrode was stored in a glass tube containing $0.1 \mathrm{M}$ TBAX in PC, fitted with a frit at one end. 
(a)

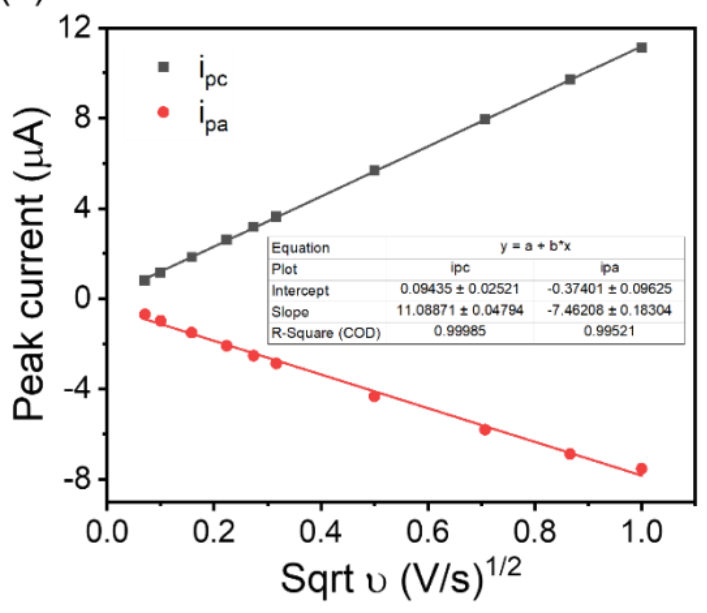

(b)

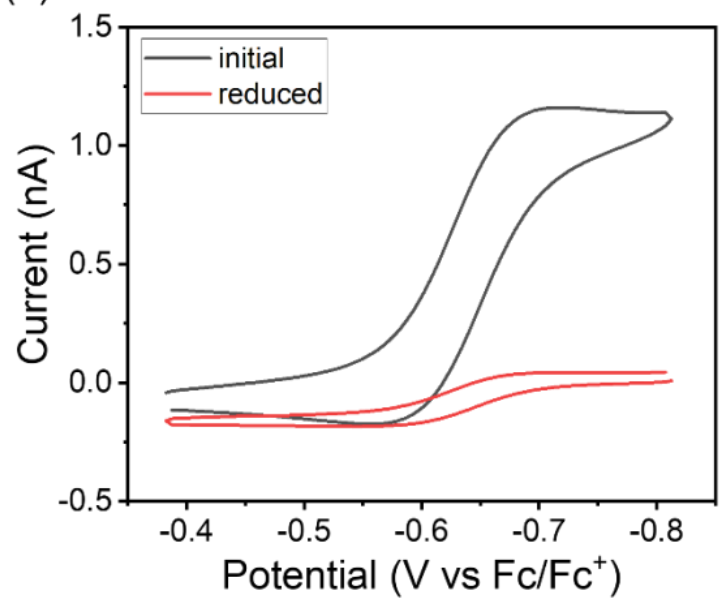

Figure S12. Randles-Sevcik analysis and cyclic voltammograms obtained for DTPV ${ }^{2+}-\mathbf{P F}_{6}{ }^{-}$using a platinum ultramicroelectrode (UME) prior to charging and immediately following the charging process. (a) Randles-Sevcik analysis of peak currents from Figure 3a (main text). (b) Bulk electrolysis characterization of the initial and reduced $\mathbf{D T P V}{ }^{2+} \mathbf{P F}_{\mathbf{6}}{ }^{-}$with Pt-UME (12.5 $\mu$ m radius) at a sweep rate of $50 \mathrm{mV} / \mathrm{s}$. UME voltammograms reveal that the charged species produces significantly lower amounts of steady-state current compared to the uncharged species. We posit that the precipitated reduced particles block some of the UME surface, effectively reducing the surface area available for the electron transfer and resulting in a lower current. Additionally, the decrease in current occurs as the precipitation of the reduced species lowers the concentration of the solution species that are sampled by the UME. 


\section{S.6 Supplementary ECSTM-BJ Experimental Data with Gate Potential}

(a)

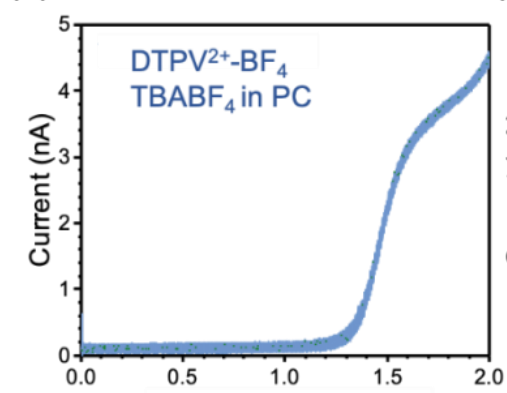

(d)

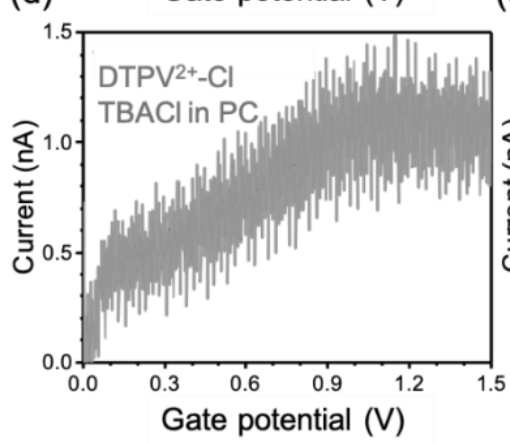

(b)

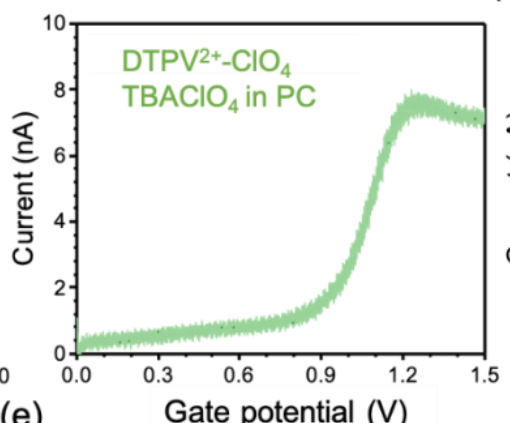

(e) (c)

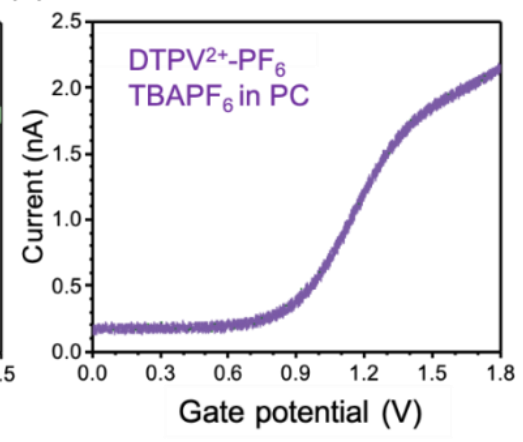

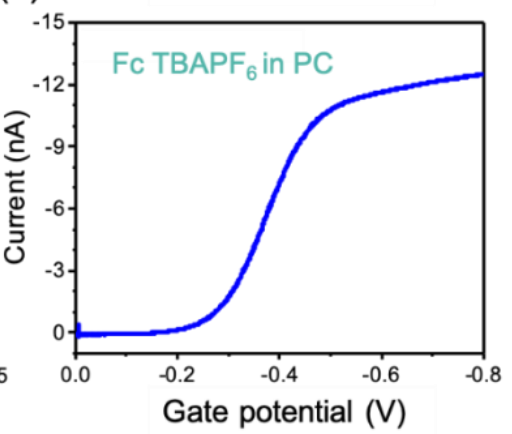

Figure S13. Linear sweep voltammetry (LSV) of $1 \mathrm{mM} \mathrm{DTPV}^{2+}-\mathbf{X}^{-}$in propylene carbonate with 0.1 M tetrabutylammonium salts of the corresponding counterion X as the electrolyte. LSV data was recorded in the STM-BJ setup used for the electrochemical conductance measurement. The current was collected and recorded with a gold tip coated by the Apiezon wax, and the gate potential was swept by a Pt electrode relative to the gold substrate electrode for $500 \mathrm{mV} / \mathrm{s}$. The oxidation of ferrocene (d) was observed at a negative gate potential with a half peak potential of $-0.4 \mathrm{~V}$, which is consistent with prior value reported in the similar setup ${ }^{6}$. The reduction of DTPV $^{2+}-\mathbf{X}^{-}$was observed at positive gate potentials. Here, only the first reduction from the dicationic state $\mathbf{D T P V}^{2+}$ to the radical cation state DTPV ${ }^{+}$was studied. The half peak potentials $\mathrm{E}_{\mathrm{p} / 2}$ in the STM-BJ system are around (a) $1.45 \mathrm{~V}$ for $\mathbf{D T P V}^{2+}-\mathbf{B F}_{4}{ }^{-}$with $\mathrm{TBABF}_{4}$; (b) $1.1 \mathrm{~V}$ for DTPV $^{2+} \mathbf{C l O}_{4}^{-}$with $\mathrm{TBAClO}_{4}$; (c) $1.2 \mathrm{~V}$ for $\mathbf{D T P V}^{\mathbf{2 +}} \mathbf{P F}_{6}{ }^{-}$with $\mathrm{TBAPF}_{6}$; (d) $0.6 \mathrm{~V}$ for $\mathbf{D T P V}^{\mathbf{2 +}}$ $\mathrm{Cl}^{-}$with TBACl; (e) $0.41 \mathrm{~V}$ for Ferrocene. 

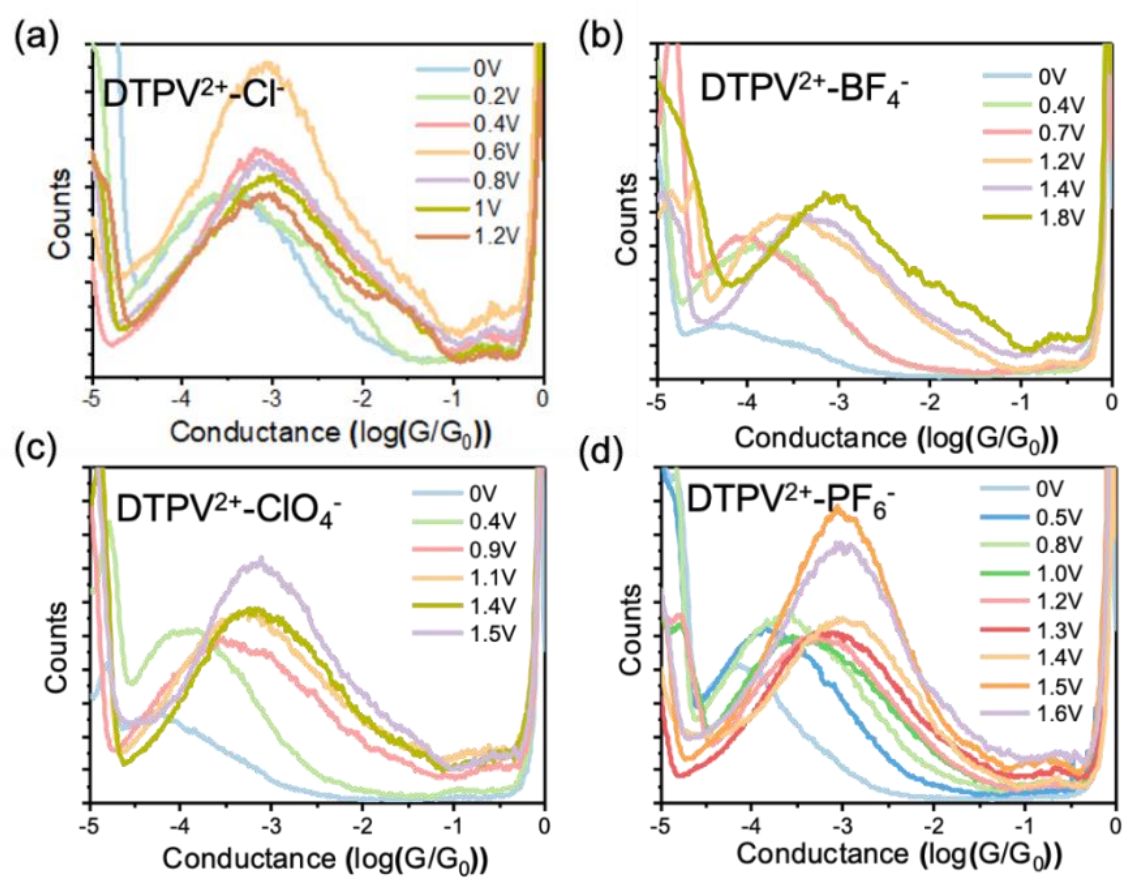

Figure S14. Logarithm-binned 1D conductance histograms for $1 \mathrm{mM} \mathrm{DTPV}^{\mathbf{2 +}} \mathbf{X}^{-}$in propylene carbonate with $0.1 \mathrm{M}$ tetrabutylammonium salts of corresponding counterion $\mathrm{X}$ at different gate potentials. $\mathrm{X}=$ (a) $\mathrm{Cl}$; (b) $\mathrm{BF}_{4}$; (c) $\mathrm{ClO}_{4}$; (d) $\mathrm{PF}_{6}$.
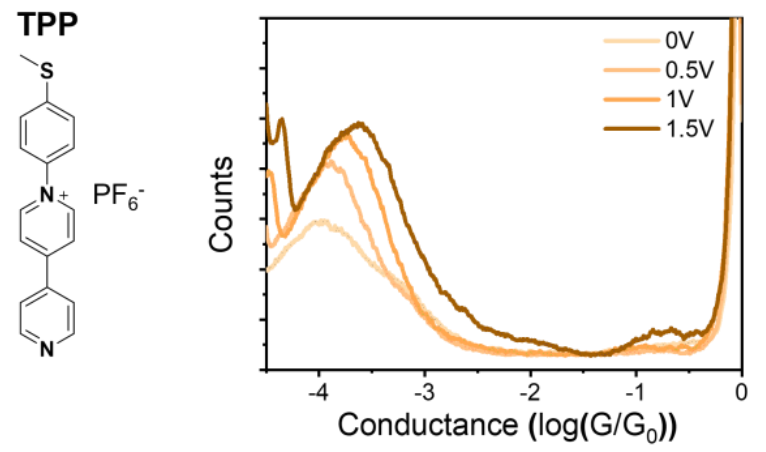

Figure S15. Logarithm-binned 1D conductance histograms for $1 \mathrm{mM}$ TPP $^{+}$PF6 $^{-}$in propylene carbonate with 0.1 M TBAPF6 at different gate potential. 


\section{S.7 Supplementary Molecular Modeling of Radical Cationic State}

S.7.1 Geometry Optimization and Electronic Structure Analysis by Density Functional Theory (DFT)

S.7.1.1 Optimized geometry in the absence of counterions in the system

Table S5. Results of optimized geometries and electronic structures of $\mathbf{D T P V}^{\mathbf{2 +}}$ and $\mathbf{D T P V}^{\cdot+}$ with the absence of counterions

\begin{tabular}{|c|c|c|c|c|}
\hline & Geometry & Dihedrals & $\begin{array}{c}\mathrm{HOMO} / \mathrm{LUMO} \\
(\mathrm{eV})\end{array}$ & $\begin{array}{c}\text { End-to-end } \\
\text { distance (Ang) }\end{array}$ \\
\hline $\mathrm{DTPV}^{2+}$ & दू & $52 / 37 / 53$ & $-8.65 /-1.61$ & 18.885 \\
\hline DTPV $^{\cdot+}$ & 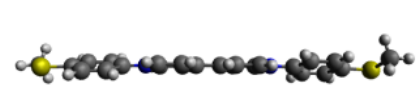 & $14 / 1 / 15$ & $-6.71(\mathrm{SOMO})$ & 19.211 \\
\hline
\end{tabular}

S.7.1.2 Conformational search of DTPV in radical cationic state with the presence of counterions in the system

It is reasonable to assume that over the timescale of electronic reduction, the two associated counterions should remain within the vicinity of the molecule. However, at longer timescales, the equilibrium state of the reduced molecule will likely only consist of a single nearby counterion provided to enforce charge neutralization. For the former case, the lowest energy geometry resulting from each ground state counterion conformational search was used to seed a geometry optimization of the reduced state in the presence of the two counterions. These results are shown in Table S6. For the latter case, the lowest energy geometry resulting from each ground state counterion conformational search was used to seed a geometry optimization of the reduced state, but with one of the neighboring counterions deleted. In all cases, substantial planarization was observed in the reduced state relative to the ground state. The results of the geometry optimizations for the reduced states are summarized in Table S6. While both geometry optimizations result in similarly planarized geometries, the addition of a counterion lowers the single occupied molecular orbital (SOMO) energy of DTPV by approximately $0.1 \mathrm{eV}$ in all cases.

Table S6. Summary of the geometry optimizations for the reduced state of DTPV adjacent to either one or two counterions

\begin{tabular}{|c|c|c|c|c|c|c|}
\hline Counterion & $\begin{array}{c}\text { Reduced ( } 2 \text { ctrion) } \\
\text { Image }\end{array}$ & $\begin{array}{c}\text { Reduced } \\
\text { (2 ctrion) } \\
\text { SOMO } \\
(\mathrm{eV})\end{array}$ & $\begin{array}{l}\text { Reduced } \\
\text { ( } 2 \text { ctrion) } \\
\text { Dihedrals }\end{array}$ & $\begin{array}{c}\text { Reduced (1 ctrion) } \\
\text { Image }\end{array}$ & $\begin{array}{c}\text { Reduced } \\
\text { (1 ctrion) } \\
\text { SOMO } \\
(\mathrm{eV})\end{array}$ & $\begin{array}{c}\text { Reduced } \\
\text { (1 ctrion) } \\
\text { Dihedral } \\
\mathrm{s}\end{array}$ \\
\hline $\mathrm{Cl}^{-}$ & & -6.28 & $40 / 3 / 47$ & & -6.35 & $43 / 4 / 46$ \\
\hline
\end{tabular}




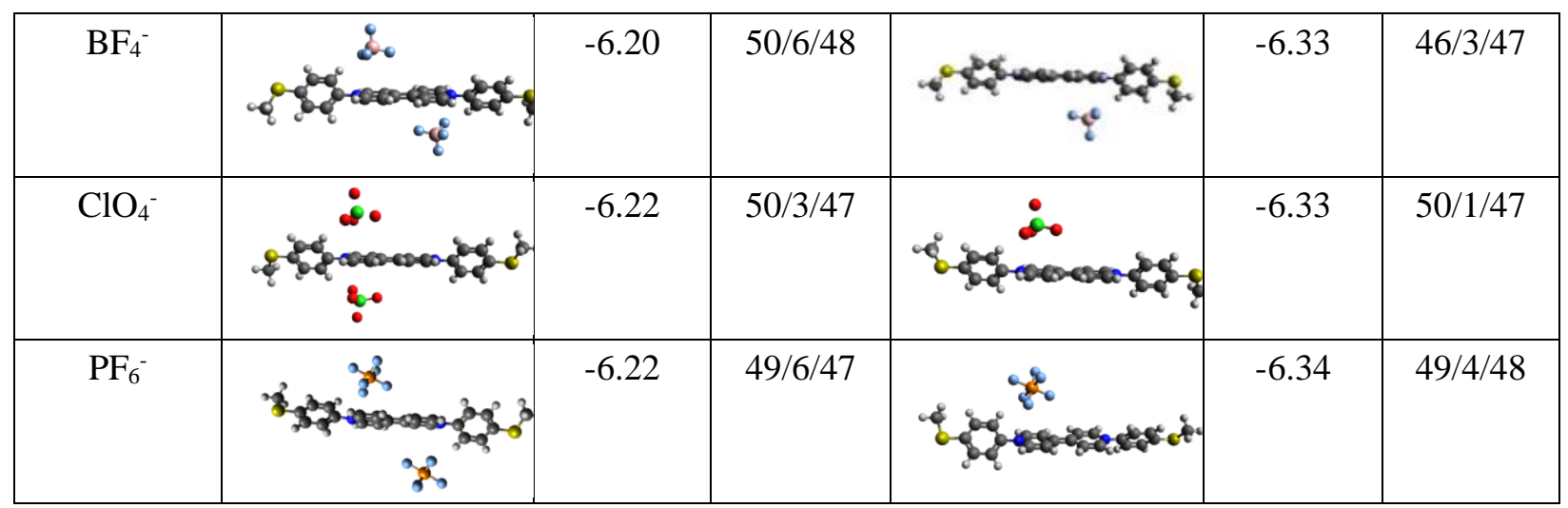

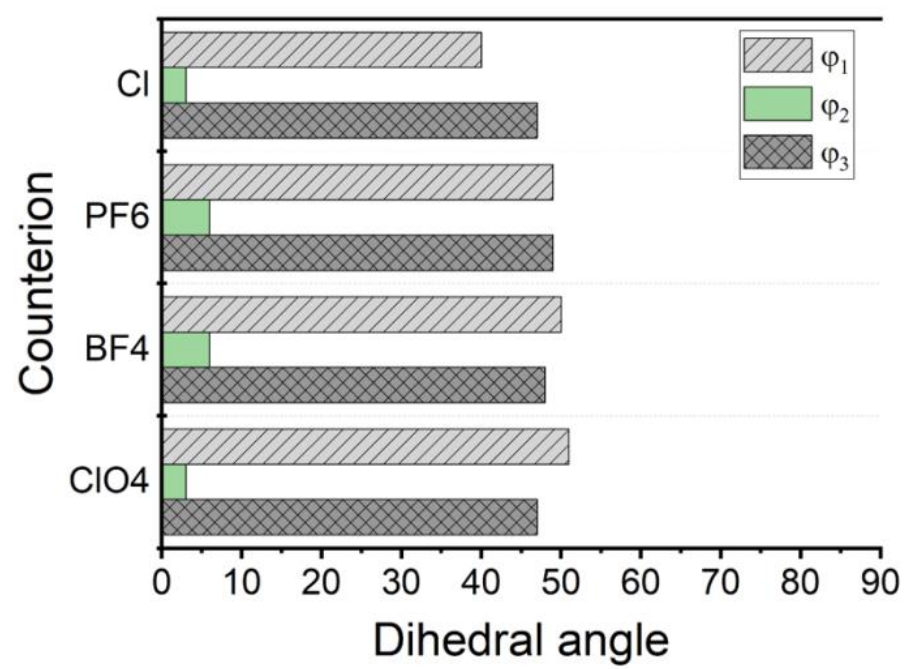

Figure S16. Torsion angles/dihedral angles between four planes of the optimized DTPV ${ }^{\cdot+}$ (radical cation state) geometries surrounded by two counterions. The results show that in all cases, the middle dihedral planarizes strongly. 
S.7.2 DFT-based transmission calculations

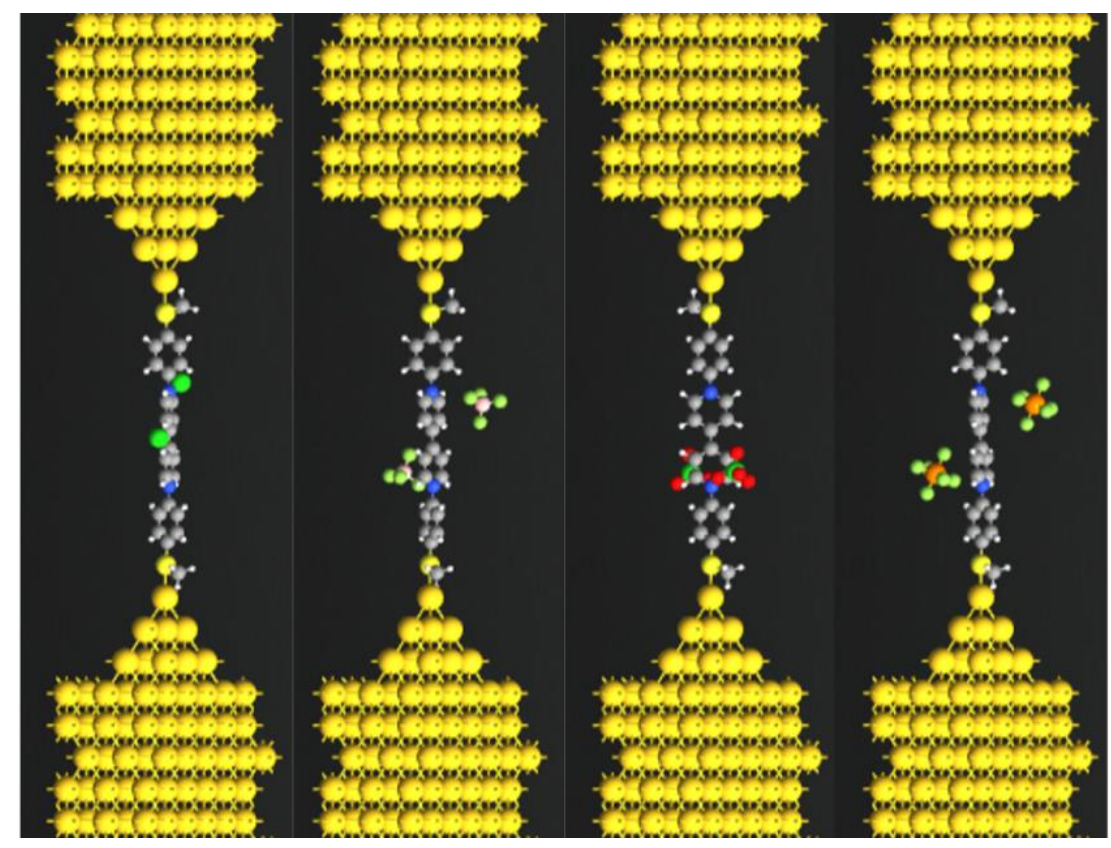

Figure S17. Molecular junctions containing $\mathbf{D T P V}^{2+}-\mathbf{X}^{-}$with the planarized conformation adopted from the optimized geometries of $\mathbf{D T P V}{ }^{\cdot+} \mathbf{X}^{-}$. From left to right, junctions are denoted as Au-

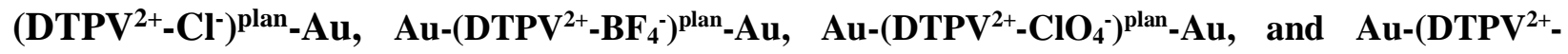
$\left.\mathbf{P F}_{6}\right)^{-)^{\text {plan }}}$-Au. 
(a)

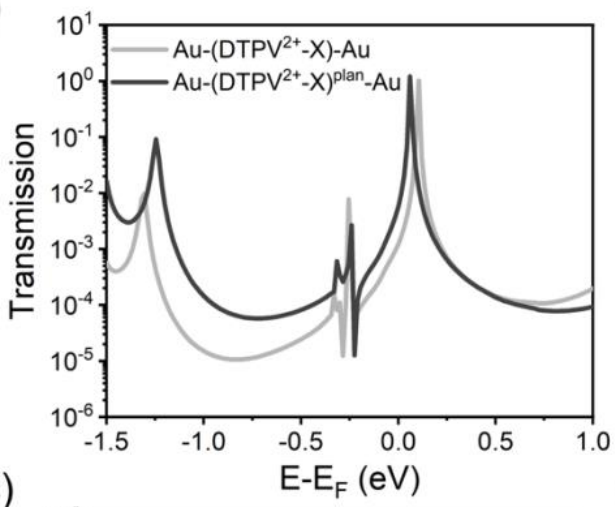

(c)

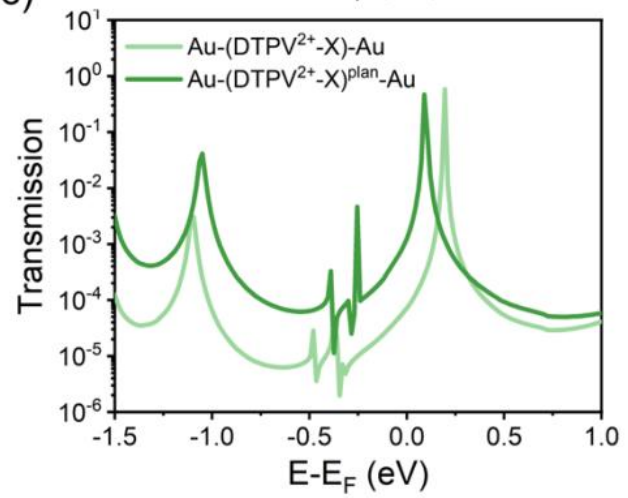

(b)

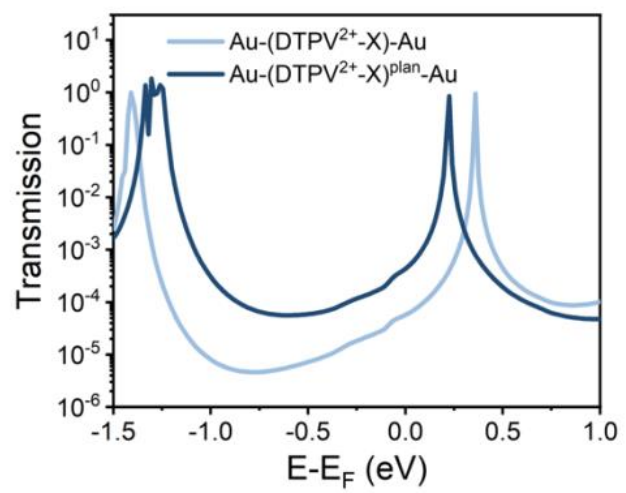

(d)

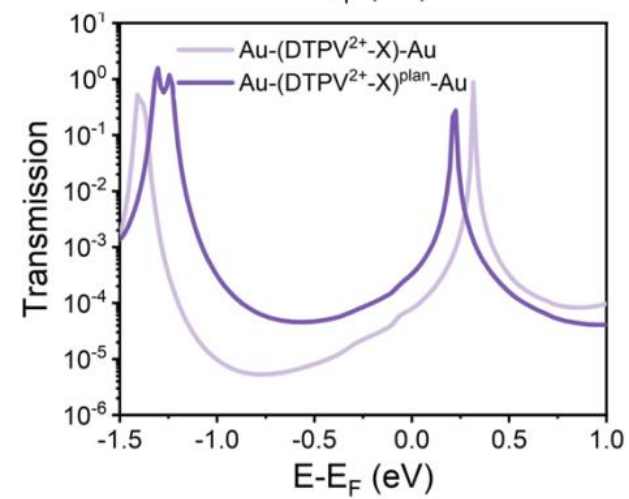

Figure S18. Transmission spectra as a function of energy for Au-(DTPV $\left.{ }^{2+}-\mathbf{X}^{-}\right)-\mathbf{A u}$ (light color) and Au-(DTPV $\left.{ }^{2+}-\mathbf{X}\right)^{\text {plan }}-\mathbf{A u}$ (dark color) with two counterions in systems. $\mathrm{E}_{\mathrm{F}}$ is the Fermi level of the junction. $\mathrm{X}=$ (a) $\mathrm{Cl}^{-}$; (b) $\mathrm{BF}_{4}^{-}$; (c) $\mathrm{ClO}_{4}^{-}$; (d) $\mathrm{PF}_{6}^{-}$. 


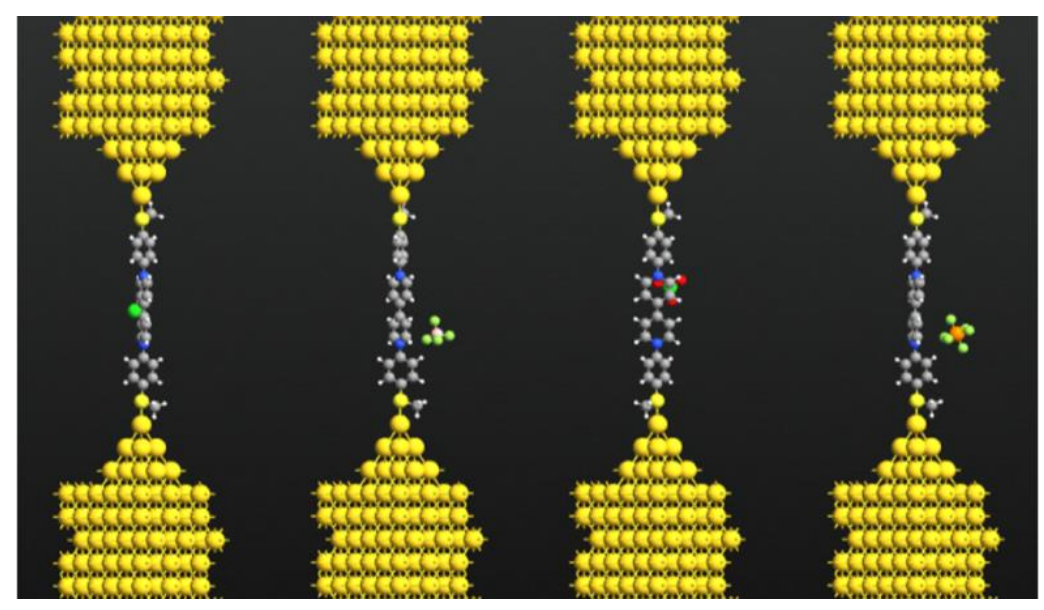

Figure S19. Molecular junctions containing $\mathbf{D T P V}^{2+} \mathbf{X}^{-}$with the planarized conformation adopted from the optimized geometries of $\mathbf{D T P V}^{\cdot+} \mathbf{- \mathbf { X } ^ { - }}$. Each system only contains on counterion. From left to right, junctions are denoted as Au-(DTPV $\left.{ }^{2+}-\mathbf{C l}^{-}\right)^{\text {plan }}-\mathbf{A u}, \mathbf{A u}-\left(\mathbf{D T P V}^{2+}-\mathbf{B F}_{4}^{-}\right)^{\text {plan }}-\mathbf{A u}, \mathbf{A u}-$ $\left(\mathrm{DTPV}^{2+}-\mathrm{ClO}_{4}^{-}\right)^{\text {plan }}-\mathrm{Au}$, and Au-(DTPV $\left.{ }^{2+}-\mathrm{PF}_{6}\right)^{\text {plan }}-\mathrm{Au}$.
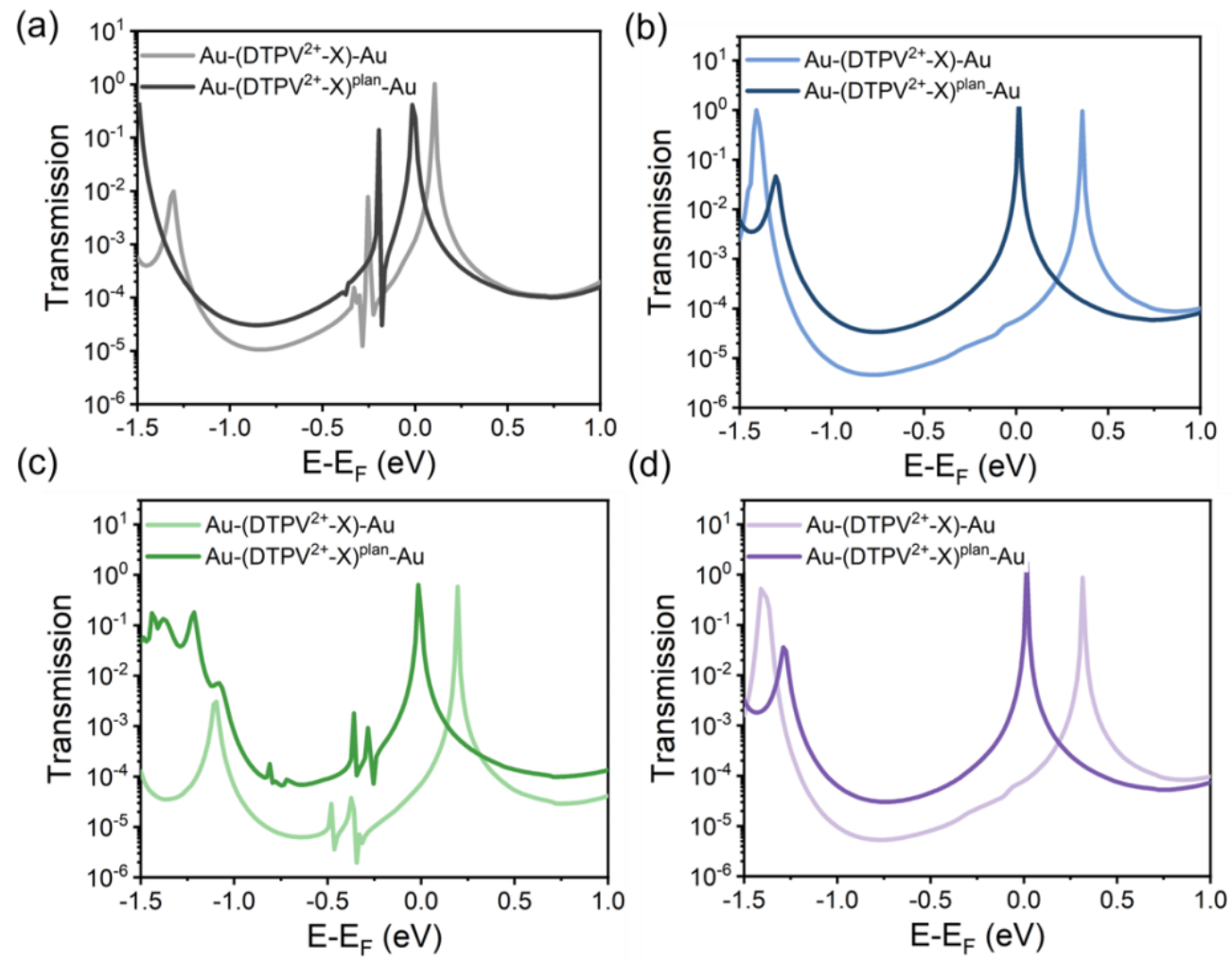

Figure S20. Transmission spectra as a function of energy for Au-(DTPV $\left.{ }^{2+}-\mathbf{X}^{-}\right)-\mathbf{A u}$ (light color) and Au-(DTPV $\left.{ }^{2+}-\mathbf{X}\right)^{\text {plan }}$-Au (dark color) with one counterion in the system. $\mathrm{E}_{\mathrm{F}}$ is the Fermi level of the junction. $\mathrm{X}=$ (a) $\mathrm{Cl}^{-}$; (b) $\mathrm{BF}_{4}^{-}$; (c) $\mathrm{ClO}_{4}^{-}$; (d) $\mathrm{PF}_{6}$. 


\section{S.8 Supplementary Junction Stability Analysis}
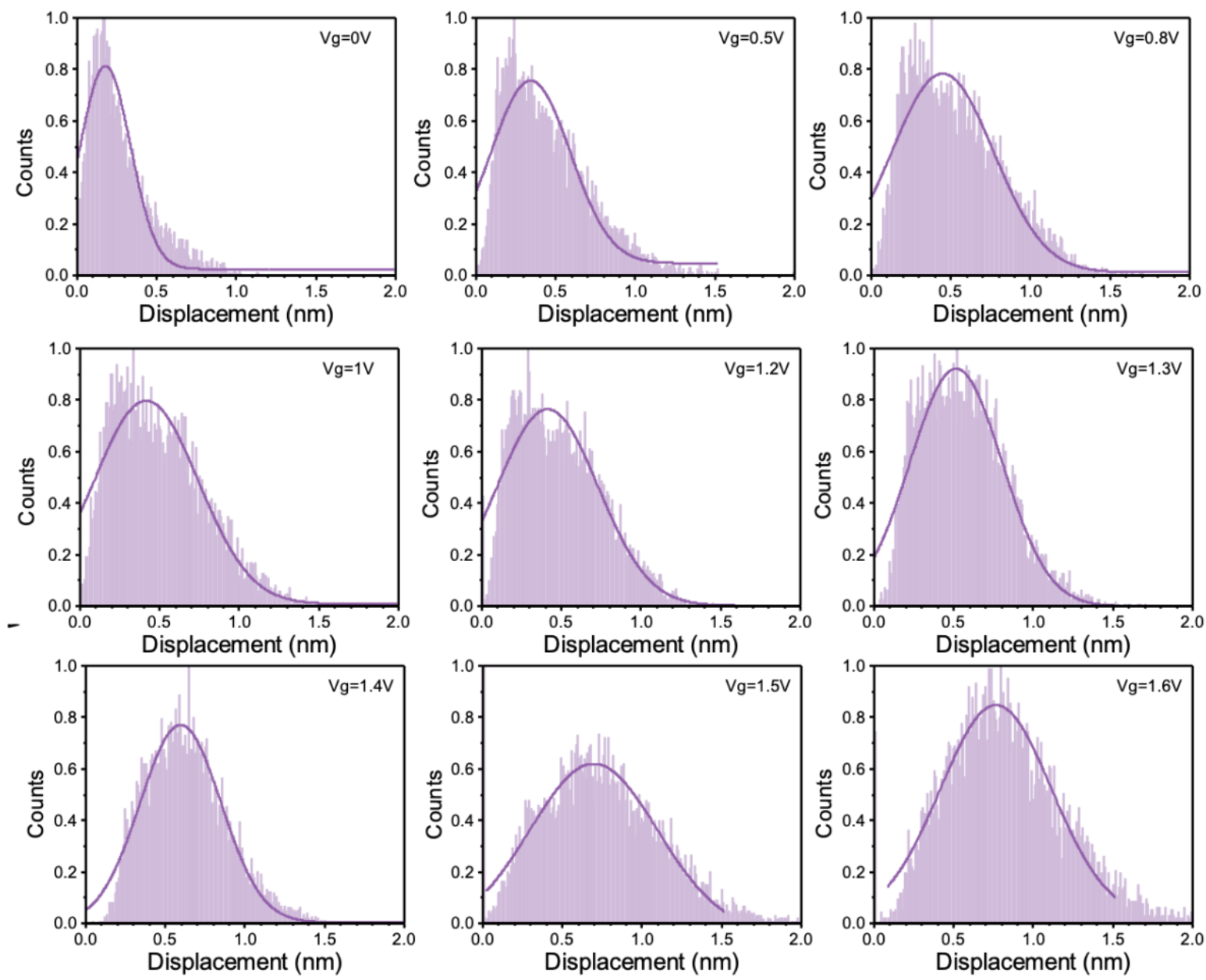

Figure S21. Logarithm-binned 1D molecular junction displacement histograms for $1 \mathrm{mM}$ DTPV $^{2+} \mathbf{P F}_{6}$ in propylene carbonate with $0.1 \mathrm{M} \mathrm{TBAPF}_{6}$ at different gate potentials. Molecular junction displacement was determined using a method described in our previous work ${ }^{7}$. In brief, the junction displacement of each single molecule conductance trace was determined by calculating the relative distance between tip-substrate displacement at a molecular conductance of $0.5 \mathrm{G}_{0}$ (corresponding to a stretched molecular junction) and the conductance corresponding to $10 \%$ of the average junction conductance calculated from 1D histograms (corresponding to the junction break point $)^{8}$. The logarithm-binned 1D displacement histograms were then fitted with the Gaussian function to determine the average molecular junction displacement from the peak values. 
(a)

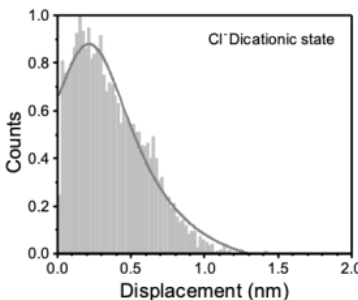

(c)

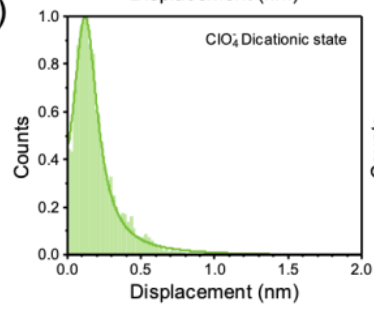

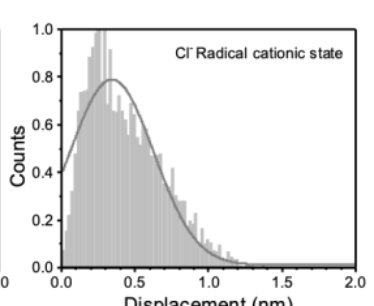

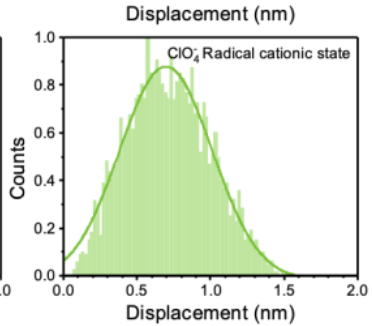

(b)

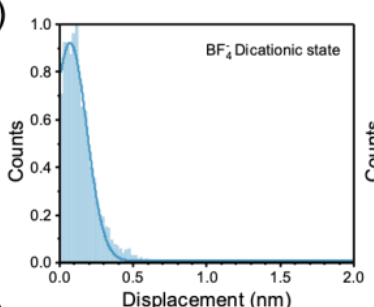

(d)

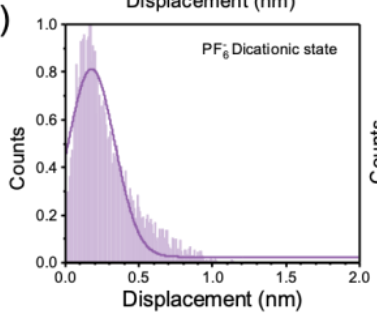

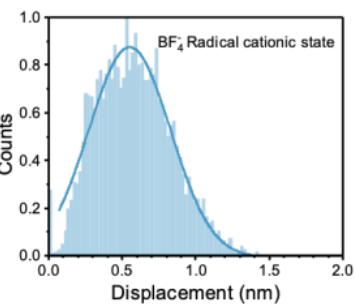

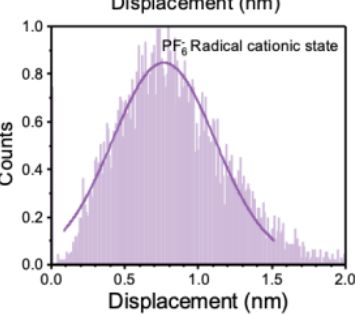

Figure S22. Comparison of logarithm-binned 1D molecular junction displacement histograms fitted with Gaussian functions for $1 \mathrm{mM} \mathrm{DTPV}^{\mathbf{2}}-\mathbf{X}$ at dicationic (2+) state and the radical cation $(++)$ state in propylene carbonate with $0.1 \mathrm{M}$ TBAX. The junction displacement of the dicationic state was determined from the single molecule conductance measurement at $0 \mathrm{~V}$ gate potential, whereas the junction displacement of the reduced radical cation state was calculated from the measurement at an overpotential of $0.4 \mathrm{~V}$ relative to the half peak potential $\left(\eta=\left|\mathrm{V}_{\mathrm{g}}-\mathrm{E}_{\mathrm{p} / 2}\right|\right)$. (a) DTPV $^{2+}{ }_{-} \mathbf{C l}^{-}$with TBACl; (b) DTPV ${ }^{2+}-\mathbf{B F}_{4}^{-}$with $\mathrm{TBABF}_{4}$; (c) DTPV $^{\mathbf{2 +}} \mathbf{C l O}_{4}^{-}$with $\mathrm{TBAClO}_{4}$; (d) DTPV $^{2+}{ }^{2+}$ F $_{6}$ - with $\mathrm{TBAPF}_{6}$. Average molecular displacement values in Figure $4 \mathrm{~b}$ (main text) were determined by fitting a Gaussian function to 1D junction displacement histograms.

(a)

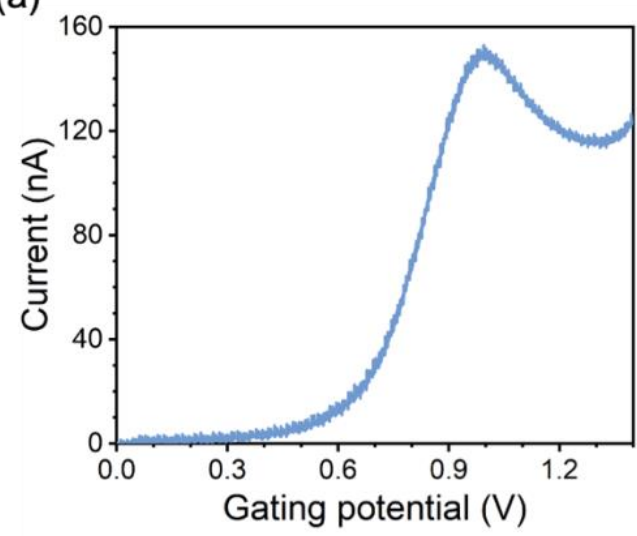

(b)

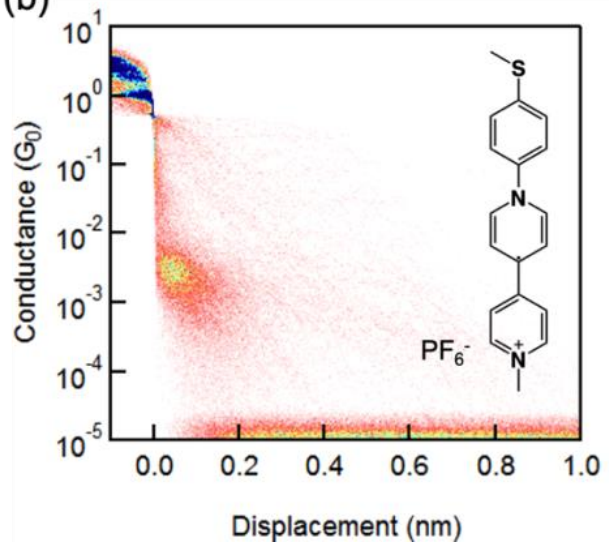

Figure S23. (a) Linear sweep voltammetry (LSV) of $1 \mathrm{mM} \mathrm{TPV}^{\mathbf{2 +}}-\mathbf{P F}_{6}{ }^{-}$in propylene carbonate with $0.1 \mathrm{M} \mathrm{TBAPF}_{6}$. (b) $2 \mathrm{D}$ conductance-displacement histograms for $\mathbf{T P V}^{\mathbf{2 +}} \mathbf{- \mathbf { P F } _ { 6 }}$ - at a gate potential of $1.2 \mathrm{~V}$. 
(a)

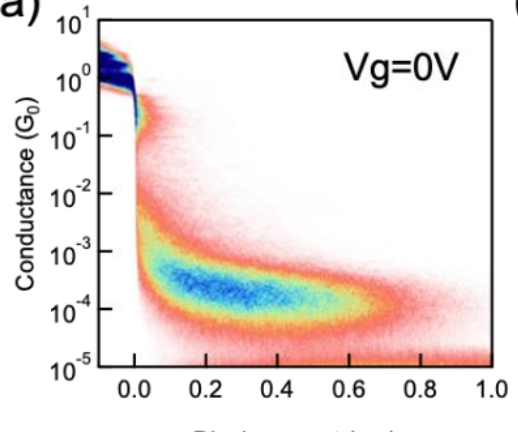

(c)

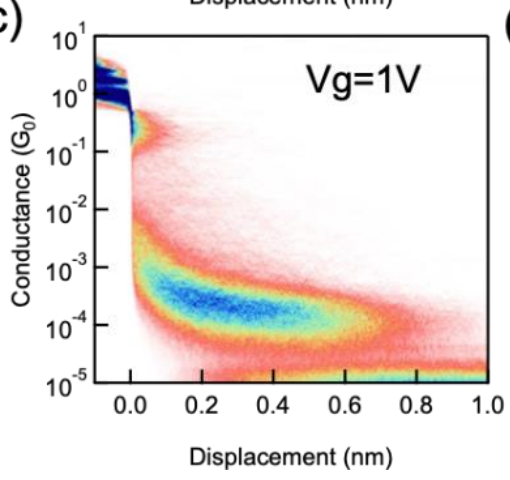

(b)

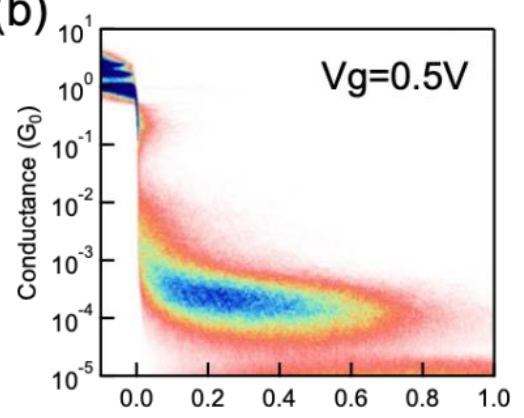

Displacement (nm)

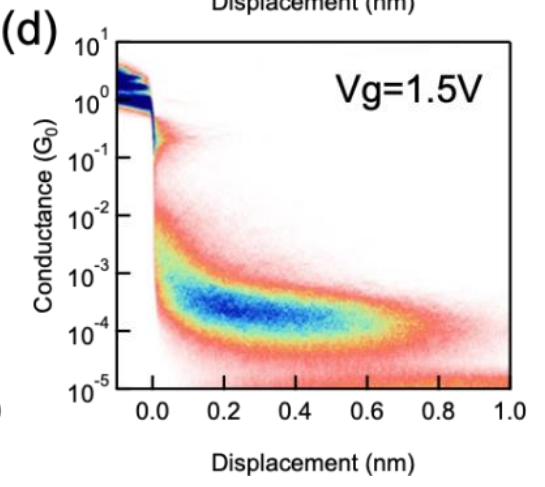

Figure S24. 2D conductance-displacement histograms for $0.1 \mathrm{mM}$ DTP with $50 \mathrm{mM} \mathrm{TBAPF}_{6}$ in PC at a gate potential of (a) $0 \mathrm{~V}$; (b) $0.5 \mathrm{~V}$; (c) $1 \mathrm{~V}$; (d) $1.5 \mathrm{~V}$. 


\section{References}

1. Zhang, W.; Gan, S.; Vezzoli, A.; Davidson, R. J.; Milan, D. C.; Luzyanin, K. V.; Higgins, S. J.; Nichols, R. J.; Beeby, A.; Low, P. J. et al. Single-Molecule Conductance of ViologenCucurbit[8]uril Host-Guest Complexes. ACS Nano 2016, 10, 5212-5020.

2. Roy, I.; Bobbala, S.; Zhou, J.; Nguyen, M. T.; Nalluri, S. K. M.; Wu, Y.; Ferris, D. P.; Scott, E. A.; Wasielewski, M. R.; Stoddart, J. F. ExTzBox: A Glowing Cyclophane for Live-Cell Imaging. J Am Chem Soc 2018, 140, 7206-7212.

3. Joseph, Y.; P, A.; Chen, X.; Michl, J.; Vossmeyer, T.; Yasuda, A. Vapor Sensitivity of Networked Gold Nanoparticle Chemiresistors: Importance of Flexibility and Resistivity of the Interlinkage. J. Phys. Chem. C 2007, 111, 12855-12859.

4. Biedermann, F.; Scherman, O. A. Cucurbit[8]uril mediated donor-acceptor ternary complexes: a model system for studying charge-transfer interactions. J. Phys. Chem. B 2012, 116, 2842-2849. 5. Rezende, M. C.; Ponce, I.; Oñate, R.; Almodovar, I.; Aliaga, C. Change of mechanism with a change of substituents for a Zincke reaction. Tetrahedron Lett. 2014, 55, 3097-3099.

6. Yin, X.; Z, Y.; Zhu, L.; Low, J. Z.; Liu, Z. F.; Cui, J.; Neaton, J. B.; Venkataraman, L.; Campos, L.M. A reversible single-molecule switch based on activated antiaromaticity. Sci. Adv. 2017, 3.

7. Li, S.; Li, J.; Yu, H.; Pudar, S.; Li, B.; Rodríguez-López, J.; Moore, J. S.; Schroeder, C. M. Characterizing intermolecular interactions in redox-active pyridinium-based molecular junctions. J. Electroanal. Chem 2020, 875.

8. Magyarkuti, A.; Adak, O.; Halbritter, A.; Venkataraman, L. Electronic and mechanical characteristics of stacked dimer molecular junctions. Nanoscale 2018, 10, 3362-3368. 